\title{
Symmetry-related transport on a fractional quantum Hall edge
}

\author{
Jinhong Park \\ Department of Condensed Matter Physics, Weizmann Institute of Science, Rehovot 76100, Israel \\ and Institute for Theoretical Physics, University of Cologne, Zülpicher Straße 77, 50937 Köln, Germany \\ Bernd Rosenow \\ Institut für Theoretische Physik, Universität Leipzig, D-04103 Leipzig, Germany \\ and Department of Condensed Matter Physics, Weizmann Institute of Science, Rehovot 76100, Israel \\ Yuval Gefen \\ Department of Condensed Matter Physics, Weizmann Institute of Science, Rehovot 76100, Israel
}

(Received 10 April 2020; revised 31 December 2020; accepted 24 March 2021; published 29 April 2021)

\begin{abstract}
Low-energy transport in quantum Hall states is carried through edge modes and is dictated by bulk topological invariants and possibly microscopic Boltzmann kinetics at the edge. Here, we show how the presence or breaking of symmetries of the edge Hamiltonian underlie transport properties, specifically dc conductance and noise. We demonstrate this through the analysis of hole-conjugate states of the quantum Hall effect, specifically the $v=2 / 3$ case in a quantum point-contact geometry. We identify two symmetries, a continuous SU(3) symmetry and a discrete $Z_{3}$ symmetry, whose presence or absence (different symmetry scenarios) dictate qualitatively different types of behavior of conductance and shot noise. While recent measurements are consistent with one of these symmetry scenarios, others can be realized in future experiments.
\end{abstract}

DOI: 10.1103/PhysRevResearch.3.023083

\section{INTRODUCTION}

\section{A. Questions we address}

The edge of quantum Hall $(\mathrm{QH})$ phases supports gapless excitations. These are responsible for low-energy dynamics in such systems, including electrical and thermal transport and noise. A convenient working framework to study this physics is to describe the edge in terms of one-dimensional chiral Luttinger modes [1]. This simple picture has proven more complex and exotic than first anticipated, especially in the context of fractional quantum Hall $(\mathrm{FQH})$ phases [2-15]. This is a particularly pressing issue when it comes to multimode edges, e.g., the case of hole-conjugate states $(1 / 2<v<1)$, where counterpropagating chiral modes are present. Varying the confining potential at the edge and accounting for the effective electrostatic and exchange interaction may give rise to edge reconstruction [16-18], where additional chiral edge modes emerge [19-26]. Topological numbers of the bulk phase impose constraints on these emergent modes, importantly, that the difference between the number of upstream and downstream chiral modes (proportional to the topological heat conductance [27]) remains invariant under edge reconstruction.

Published by the American Physical Society under the terms of the Creative Commons Attribution 4.0 International license. Further distribution of this work must maintain attribution to the author(s) and the published article's title, journal citation, and DOI.
The interplay of disorder and interactions at the edge may lead to renormalization of the edge modes. One paradigmatic example is the edge of a bulk filling factor $v_{\text {bulk }}=2 / 3$, where a downstream charge $2 / 3$ mode and an upstream neutral mode may emerge [28-32]. At the stable fixed point the charge and neutral sectors are decoupled; underlying the latter is a $\mathrm{SU}(2)$ symmetry, related to the fact that neutral excitations (neutralons) may be mapped onto spin- $1 / 2$ fermions.

The Kane-Fisher-Polchinski model [28] serves as an example of an emergent symmetry [SU(2)] due to a renormalization group (RG) of edge modes. This evokes questions of much broader scope: What is the interplay of topology and emergent symmetry, and what is its role in dictating transport and noise at the edge? To address these questions, we focus here on a paradigmatic example, that of a reconstructed and renormalized edge of the $v=2 / 3 \mathrm{FQH}$ phase. Indeed, the predicted upstream neutral mode propagation [28] has been observed experimentally [2-7,33]. Subsequent experiments $[11,13,14,34]$ implementing quantum point contacts (QPCs) led to the conclusion that the original picture of the edge $[19,21,28]$ needed to be modified [20]. Following a RG analysis of the reconstructed edge [Fig. 1(a)], the emerging picture comprises an intermediate fixed point with two downstream running charged modes and two upstream neutral modes [Fig. 1(b)]. While many of the available experimental data are compatible with this edge structure (e.g., the conductance plateau of $e^{2} / 3 h$ and the existence of upstream modes), the observation of significant shot noise (corresponding to the Fano factor $2 e / 3)$ on the conductance plateau $[11,13,34]$ is still puzzling. The conductance plateau implies that each of 


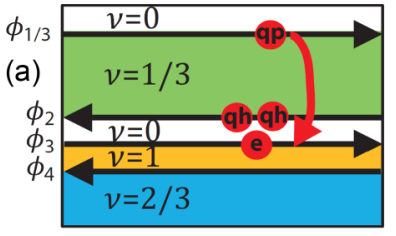

(b)

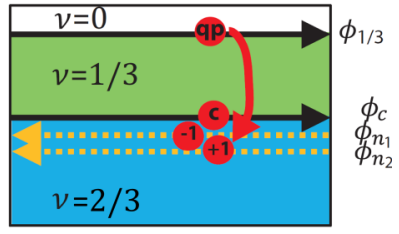

FIG. 1. Edge structures and excitations of the bulk filling factor $v_{\text {bulk }}=2 / 3$. (a) Four bare edge modes are depicted with arrowheads indicating the direction of each mode [downstream (right movers) or upstream (left movers)]. A quasiparticle tunneling from the outermost edge mode to the inner ones turns into two quasiholes and one electron. Due to the presence of a vacuum region with $v=0$, only electrons can tunnel to mode $\phi_{4}$, leaving the fractional charge on $\phi_{4}$ invariant and giving rise to a $Z_{3}$ symmetry between sectors with fractional charge $0,1 / 3$, and $2 / 3$. (b) Due to electron tunneling between the three inner edge modes $\left(\phi_{2}, \phi_{3}\right.$, and $\left.\phi_{4}\right)$ and the bare interaction between them, the inner three edge modes are renormalized to a downstream charge mode (denoted as a black solid line) with the filling factor discontinuity $\delta v=1 / 3$ and two upstream neutral modes (denoted as yellow dashed lines). The same tunneling process as in (a) now creates a chargeon and neutral charges $(-1,1)$.

the charge modes either fully reflected or fully transmitted. Such a scenario would suggest the absence of shot noise on the plateau [35]. Here, we identify a scenario with a broken discrete $Z_{3}$ symmetry and a statistically preserved SU(3) symmetry, in which a quantized conductance $e^{2} / 3 h$ and a $2 e / 3$ Fano factor are realized, in agreement with recent experimental findings $[11,13,34]$. We also consider different symmetry scenarios which can be investigated in future experiments.

Moreover the robustness of (and evidence for) incoherent transport [8,12,30-32,36-38], described by microscopic Boltzmann kinetics of edge modes, underlies most experimental studies. Further experimental evidence of coherent upstream propagation [31] of neutral modes (underlain by intriguing hidden symmetries as discussed below) is needed.

It is clearly desirable to incorporate our current understanding of edge structure (dictated by topological constraints) and edge dynamics within a general symmetry based framework. This is the main goal of this paper. While we focus here on the $v=2 / 3$ reconstructed and renormalized edge, the ideas presented here can serve as guidelines in studying other topological states. Furthermore, our study may motivate the search for various symmetry scenarios with new experimental manifestations.

\section{B. Summary of main results}

This paper demonstrates that given the topological invariants of the bulk phase (dictated by the filling factor), different symmetries of the edge modes may underlie qualitatively different transport behavior, specifically the dc conductance and the low-frequency nonequilibrium noise. This facilitates the engineering of experimentally controlled setups (of the same bulk phase) with designed, symmetry-related, behavior. In order to demonstrate our approach, we consider a specific geometry, write down the most general fixed point action compatible with this setup, identify the relevant symmetries, and show how their presence (or absence) determines the resulting transport properties.
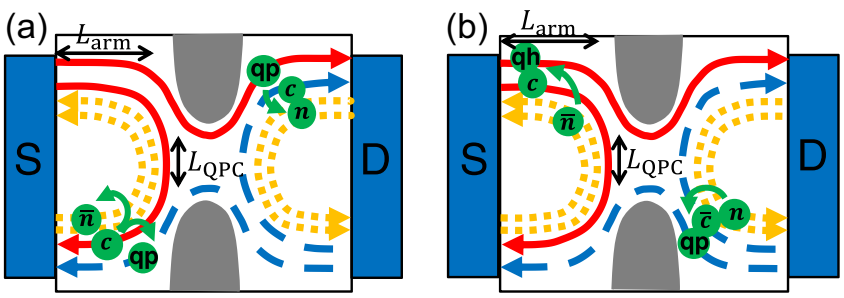

FIG. 2. A sketch of a two-step mechanism contributing to the conductance in a two-terminal setup with a quantum point contact (QPC). Potentials are tuned such that the outermost mode is fully transmitted through the QPC, while the inner ones are fully reflected. The outer charge mode supports quasiparticles (qp) and quasiholes (qh), while the inner one supports chargeons $(c)$ and antichargeons $(\bar{c})$, all four carrying a charge of $\pm e / 3$. Similarly, the two neutral modes support neutral excitations, neutralons $(n)$ or antineutralons $(\bar{n})$. The size of the QPC region and the length of the arms between the contacts and the QPC are $L_{\mathrm{QPC}}$ and $L_{\mathrm{arm}}$, respectively. (a) Equilibration processes involving charge tunneling and the creation of neutralons or antineutralons are $\mathrm{qp} \rightarrow c+n$ (upper right corner) or $c \rightarrow \mathrm{qp}+\bar{n}$ (lower left corner) represented in Eq. (10), with $\ell_{\text {ch-eq, } 0} \ll L_{\text {arm }}$. (b) The excited (anti)neutralons arrive at the lower right (upper left) corner, and they decay giving rise to qp- $\bar{c}$ (qh-c) pairs. The excited qp, qh, c, or $\bar{c}$ flow to the drain $\mathrm{D}$, contributing to the conductance. In the presence of $H_{n \bar{n}}$ [Eq. (13)], mixing between neutralons and antineutralons occurs over a distance $\ell_{n \bar{n}, 0} \ll L_{\mathrm{QPC}}$.

We start by identifying two symmetries of the $v=2 / 3$ edge, whose presence or absence (different symmetry scenarios) dictate qualitatively different types of behavior of the conductance and the shot noise: a continuous SU(3) symmetry and a discrete $Z_{3}$ symmetry. The former symmetry acts in the subspace spanned by the neutral modes. Inspired by highenergy terminology, we refer to the basic neutral excitations as up, down, and strange [cf. Eq. (6) and the discussion thereafter]. The space of these neutralons forms the fundamental representation of an $\mathrm{SU}(3)$ symmetry group. The conjugate representation is associated with antineutralons. At the fixed point, the action is invariant under SU(3) operations; as discussed below, one may break this symmetry. If the symmetry breaking that occurs is spatially randomly varying and selfaveraging, one can refer to a "statistically preserved" SU(3) symmetry. In physical terms, the breaking of the SU(3) symmetry takes place through charge equilibration in the charge sector of the chiral modes [see Fig. 1(b)]. The second important group here is a discrete $Z_{3}$ symmetry [cf. Eq. (13)], related to three different sectors of the charge on $\phi_{4}$ with the fractional charge $0,1 / 3$, or $2 / 3$. This group is not to be confused with the $Z_{3}$ subgroup of the $\mathrm{SU}(3)$ symmetry. Breaking this $Z_{3}$ symmetry allows us to connect the neutralon sector with the antineutralon sector. This may be achieved by the tunneling of $1 / 3$ charges into the innermost bare edge mode [cf. Fig. 1(a)].

To see how the symmetries affect transport behavior in a specific configuration, we consider a two-terminal setup (cf. Fig. 2) with a QPC. The edge of the system consists of the edge modes depicted in Fig. 1(b). The QPC is tuned such that the outermost charge mode is fully transmitted while the inner ones are fully reflected, naively leading to a quantized conductance of $(1 / 3)\left(e^{2} / h\right)$. Through charge equilibration 
TABLE I. A summary of experimental manifestations of two symmetries, a continuous one, SU(3), and a discrete threefold one, $Z_{3}$ [cf. Eq. (12)]. Model A (model B) corresponds to the cell in the first (second) row and the third column.

\begin{tabular}{|c|c|c|c|}
\hline & Exact SU(3) symmetry & $\ell_{\text {ch-eq }, 0} \gg L_{\text {arm }}$ & $\ell_{\text {ch-eq }, 0} \ll L_{\text {arm }}$ \\
\hline Conserved $Z_{3}$ symmetry & $\begin{array}{l}\text { Conductance plateau } e^{2} /(3 h) \\
\quad \text { zero noise on the plateau }\end{array}$ & $\begin{array}{c}\text { Mesoscopic conductance fluctuation; } \\
G=e^{2}(1-R) /(3 h), S_{\mathrm{D}}=2 e^{3} R V_{0} /(9 h)\end{array}$ & $\begin{array}{c}\text { Zero conductance; } \\
\text { zero noise }\end{array}$ \\
\hline Broken $Z_{3}$ symmetry & & Conductance plateau $e^{2} /(3 h)$ & Conductance plateau $e^{2} /(3 h)$; \\
\hline$\left(\ell_{n \bar{n}, 0} \ll L_{\mathrm{QPC}}\right)$ & & nonuniversal noise & Fano factor of $2 e / 3$ \\
\hline
\end{tabular}

between the charge modes [cf. Fig. 2(a), upper right] that breaks the SU(3) symmetry of the fixed point, only one type of neutral excitation (neutralons, but not antineutralons) is created. These neutralons then decay, converting into quasiparticles in the outermost mode and quasiholes in the inner charge mode [cf. Fig. 2(b), lower right], generating an additional current at drain $\mathrm{D}$, and hence undermining conductance quantization (cf. the upper row in Table I). Breaking the $Z_{3}$ symmetry gives rise to the emergence of a quantized conductance, $(1 / 3)\left(e^{2} / h\right)$. The reason is that with the $Z_{3}$ symmetry broken, the generation of neutralons and antineutralons in the course of equilibration is equally probable, implying no additional dc current at drain $\mathrm{D}$ yet a contribution to shot noise with a quantized Fano factor, $2 e / 3$. Experimental manifestations of the various symmetry scenarios are summarized in Table I.

\section{Structure of this paper}

The outline of this paper is the following. In Sec. II we describe a $S U(3)$ fixed point emerging at intermediate energies. In Sec. III, we consider tunneling operators near the fixed point, each of which break or preserve the $\mathrm{SU}(3)$ or $Z_{3}$ symmetry, and elaborate specific models for the two-terminal setup. In Sec. IV, we analyze transport properties (dc conductance and nonequilibrium noise), demonstrating how they are affected by the presence or absence of certain symmetries. Section V is a summary.

\section{SU(3)-SYMMETRIC FIXED POINT}

To make this discussion more self-contained, in the following we give an overview of the derivation of the SU(3)- symmetric intermediate fixed point action [20] describing the inner three edge modes. We start by considering the filling fraction profile sketched in Fig. 1(a), consisting of a $v=1 / 3$ incompressible region near the boundary of the sample, separated by more narrow $v=0$ and $v=1$ regions from the bulk. The drop in the filling fraction to zero is dictated by topology, in the sense that the $1 / 3 \mathrm{FQH}$ state is topologically distinct from the $2 / 3$ state, and hence a direct transition between them is not possible. Such a filling fraction profile is compatible with all topological constraints and can in principle be stabilized by a suitably chosen external potential. Modifications making this profile more realistic are described via the inclusion of interaction and scattering terms.

Edge states arise at the boundary of two incompressible regions, such that there is an outermost downstream $1 / 3$ edge mode described by a boson field $\phi_{1 / 3}$, rather well separated from an upstream 1/3 mode described by a field $\phi_{2}$, a downstream integer mode described by $\phi_{3}$, and another upstream $1 / 3$ mode described by $\phi_{4}$. We want to focus on the inner three edge modes at the moment, and we assume that they are spatially in close proximity, much closer to each other than to the outermost $1 / 3$ mode. Due to the presence of the integer incompressible region between the two fractional modes, only electron scattering between them is possible. Although electron scattering is irrelevant in the absence of Coulomb interaction, spatially random scattering becomes relevant in the presence of sufficiently strong interactions, and the RG flows towards strong coupling for the electron tunneling [20].

In order to describe the disorder-dominated strongcoupling fixed point, one next makes an educated guess for a fixed point action, consisting of a charge mode $\phi_{c} \equiv\left(\phi_{2}+\right.$ $\left.\phi_{3}+\phi_{4}\right) / \sqrt{3}$ and two neutral modes $\phi_{n_{1}} \equiv\left(3 \phi_{2}+\phi_{3}\right) / \sqrt{2}$ and $\phi_{n_{2}} \equiv\left(\phi_{2}+\phi_{3}+2 \phi_{4}\right) / \sqrt{2}$ :

$$
\begin{aligned}
S_{0}=S_{c}+S_{n}, & \\
S_{c}=\frac{1}{4 \pi} \int d x d t\left[\partial_{x} \phi_{c}\left(-\partial_{t}-v_{c} \partial_{x}\right) \phi_{c}\right] & \\
S_{n}=\int d x d t & {\left[\frac{1}{4 \pi}\left[\partial_{x} \phi_{n_{1}}\left(\partial_{t}-v_{n} \partial_{x}\right) \phi_{n_{1}}+3 \partial_{x} \phi_{n_{2}}\left(\partial_{t}-v_{n} \partial_{x}\right) \phi_{n_{2}}\right]\right.} \\
& \left.\quad-\frac{1}{2 \pi a}\left(\xi_{1}(x) e^{i \sqrt{2} \phi_{n_{1}}}+\xi_{2}(x) e^{-i \phi_{n_{1}} / \sqrt{2}} e^{3 i \phi_{n_{2}} / \sqrt{2}}+\xi_{3}(x) e^{i \phi_{n_{1}} / \sqrt{2}} e^{3 i \phi_{n_{2}} / \sqrt{2}}+\text { H.c. }\right)\right] .
\end{aligned}
$$

The charge and neutral modes satisfy commutation relations $\left[\phi_{c}(x), \phi_{c}\left(x^{\prime}\right)\right]=i \pi \operatorname{sgn}\left(x-x^{\prime}\right), \quad\left[\phi_{n_{1}}(x), \phi_{n_{1}}\left(x^{\prime}\right)\right]=$ $-i \pi \operatorname{sgn}\left(x-x^{\prime}\right)$, and $\left[\phi_{n_{2}}(x), \phi_{n_{2}}\left(x^{\prime}\right)\right]=-i \pi \operatorname{sgn}\left(x-x^{\prime}\right) / 3$. The second line of Eq. (1) originates from electron tunneling between the inner modes. $\xi_{i=1,2,3}$ are random variables. We note that the six electron tunneling operators in the second line of Eq. (1), together with the operators $\partial_{x} \phi_{n_{1}}$ and $\partial_{x} \phi_{n_{2}}$, constitute the generators of the symmetry group SU(3), in the 
sense that their commutation relations form the $\mathrm{SU}(3)$ algebra. One can show that the action equation (1) indeed describes an attractive fixed point in the sense that all interaction terms between a charge mode and neutral modes, and among neutral modes, flow to zero.

In order to make the $\mathrm{SU}(3)$ symmetry more explicit, we next fermionize the action equation (1). The idea is to introduce an auxiliary boson which does not couple to the two neutral mode bosons $\phi_{n_{1}}, \phi_{n_{2}}$, and use the two neutral modes and the auxiliary boson to fermionize the model. Including the action for an auxiliary field $\chi$ (see Ref. [28] for a similar procedure) and performing fermionization in terms of a three-component fermion field $\Psi(x)=e^{i \chi / \sqrt{3}}\left[e^{-i\left(\phi_{n_{1}}+\phi_{n_{2}}\right) / \sqrt{2}}, e^{i\left(\phi_{n_{1}}-\phi_{n_{2}}\right) / \sqrt{2}}, e^{i \sqrt{2} \phi_{n_{2}}}\right]^{T} / \sqrt{2 \pi a}$ $=e^{i \chi / \sqrt{3}}\left[\psi_{u}, \psi_{d}, \psi_{s}\right]^{T}$, Eq. (1) reads

$$
S_{n}=\int d x d t\left\{i \Psi^{\dagger}\left(-\partial_{t}+v_{n} \partial_{x}\right) \Psi-\left[\xi_{1} \Psi^{\dagger}\left(\frac{\lambda_{1}+i \lambda_{2}}{2}\right) \Psi+\xi_{2} \Psi^{\dagger}\left(\frac{\lambda_{6}+i \lambda_{7}}{2}\right) \Psi+\xi_{3} \Psi^{\dagger}\left(\frac{\lambda_{4}+i \lambda_{5}}{2}\right) \Psi+\text { H.c. }\right]\right\} \text {. }
$$

Here, the matrices $\lambda_{1,2,4,5,6,7}$ are nondiagonal generators of the SU(3) group (see Appendix A for more details).

In the fermion picture, the tunneling corresponds to random rotations between the fermions and can be transformed away by choosing a new basis via a space-dependent SU(3) rotation. The random terms are completely eliminated via performing a SU(3) gauge transformation of $\tilde{\Psi}(x)=U(x) \Psi(x)$; the action becomes diagonal as

$$
S_{n}=i \int d x d t \tilde{\Psi}^{\dagger}\left(-\partial_{t}+v_{n} \partial_{x}\right) \tilde{\Psi}
$$

Here,

$$
U(x)=T_{x} e^{-\frac{i}{2 v_{n}} \int_{-\infty}^{x} d x^{\prime}\left[\xi_{1}\left(\lambda_{1}+i \lambda_{2}\right)+\xi_{2}\left(\lambda_{6}+i \lambda_{7}\right)+\xi_{3}\left(\lambda_{4}+i \lambda_{5}\right)+\text { H.c. }\right]}
$$

denotes the position-dependent SU(3) rotation matrix. Transforming away the disorder in this way makes the velocities all equal.

The introduction of the auxiliary boson has contributed an additional U(1) symmetry to the problem. This extra symmetry can be removed by performing a rebosonization of the rotated fermionic fields as $\tilde{\Psi}^{T}=e^{i \tilde{\chi} / \sqrt{3}}\left[\tilde{\psi}_{u}, \tilde{\psi}_{d}, \tilde{\psi}_{s}\right]$. Here,

$$
\begin{aligned}
\tilde{\psi}_{u} & \equiv \frac{1}{\sqrt{2 \pi a}} e^{-i\left(\tilde{\phi}_{n_{1}}+\tilde{\phi}_{n_{2}}\right)}, \\
\tilde{\psi}_{d} & \equiv \frac{1}{\sqrt{2 \pi a}} e^{i\left(\tilde{\phi}_{n_{1}}-\tilde{\phi}_{n_{2}}\right)}, \\
\tilde{\psi}_{s} & \equiv \frac{1}{\sqrt{2 \pi a}} e^{i \sqrt{2} \tilde{\phi}_{n_{2}}}
\end{aligned}
$$

are referred to as up, down, and strange neutralons in this paper. In this way, and omitting the tilde, Eq. (2) becomes

$$
\begin{aligned}
S_{n}=\frac{1}{4 \pi} \int d x d t & \left\{\left[\partial_{x} \phi_{n_{1}}\left(\partial_{t}-v_{n} \partial_{x}\right) \phi_{n_{1}}\right.\right. \\
& \left.\left.+3 \partial_{x} \phi_{n_{2}}\left(\partial_{t}-v_{n} \partial_{x}\right) \phi_{n_{2}}\right]\right\} .
\end{aligned}
$$

The corresponding Hamiltonian together with the Hamiltonian related to the charge modes is given by

$$
\begin{aligned}
H_{0}=H_{n}+H_{c}= & \frac{v_{n}}{4 \pi} \int d x\left[\left(\partial_{x} \phi_{n_{1}}\right)^{2}+3\left(\partial_{x} \phi_{n_{2}}\right)^{2}\right] \\
& +\frac{1}{4 \pi} \int d x\left[v_{c}\left(\partial_{x} \phi_{c}\right)^{2}+v_{1 / 3}\left(\partial_{x} \phi_{1 / 3}\right)^{2}\right] .
\end{aligned}
$$

Two representations of the $\mathrm{SU}(3)$ group are displayed in Fig. 3. In general, neutralon states, which form the fundamen- tal representation of the SU(3) group, are labeled by charges $(m, n)$ that are eigenvalues of the diagonal generators $\lambda_{3}$ and $\lambda_{8} \sqrt{3}$, respectively:

$$
\lambda_{3}|j\rangle=m|j\rangle, \quad \lambda_{8} \sqrt{3}|j\rangle=n|j\rangle,
$$

with

$$
\lambda_{3}=\left(\begin{array}{ccc}
1 & 0 & 0 \\
0 & -1 & 0 \\
0 & 0 & 0
\end{array}\right), \quad \lambda_{8}=\frac{1}{\sqrt{3}}\left(\begin{array}{ccc}
1 & 0 & 0 \\
0 & 1 & 0 \\
0 & 0 & -2
\end{array}\right)
$$

The neutralon states of Fig. 3 (depicted as red dots) are denoted as $|u\rangle=(1,0,0)^{T}=\psi_{u}^{\dagger}|0\rangle,|d\rangle=(0,1,0)^{T}=\psi_{d}^{\dagger}|0\rangle$, and $|s\rangle=(0,0,1)^{T}=\psi_{s}^{\dagger}|0\rangle$, which correspond to $(m, n)=$ $(1,1),(-1,1)$, and $(0,-2)$, respectively.

Likewise, the conjugate representation is associated with the antineutralons $(\bar{u}, \bar{d}$, and $\bar{s}$ ) (depicted as blue dots in Fig. 3). At the intermediate SU(3) fixed point, the neutralon sector is completely disconnected from the antineutralon sector. In the next section, we will show that breaking

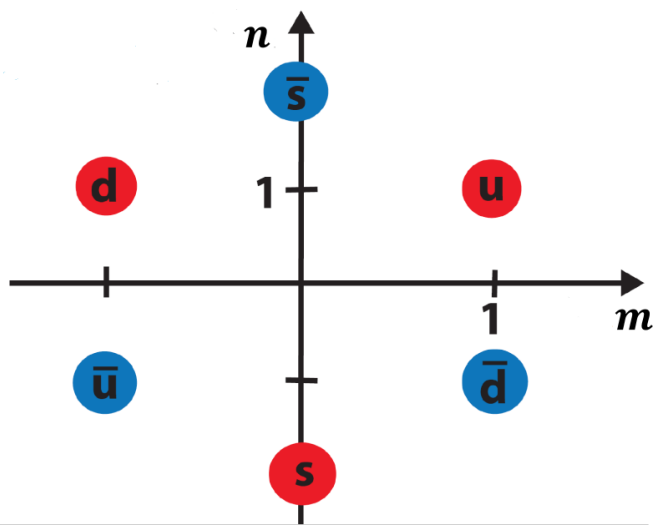

FIG. 3. SU(3) group representations. Quasiparticles described by charges $(m, n)$ [cf. Eq. (8)] form the fundamental representation of $\mathrm{SU}(3)$. In analogy to the quantum numbers $\left(I_{z}, Y\right)$ of isospin and hypercharge in flavor SU(3) [39], the elementary excitations (neutralons) are labeled $u, d$, and $s$. The corresponding antineutralons form the conjugate representation; a conversion of neutralons into antineutralons is only possible if the $Z_{3}$ symmetry is broken. This can be achieved by allowing fractional quasiparticles to tunnel into and out of the innermost edge mode. More details pertaining to the $Z_{3}$ symmetry can be found in Eq. (14) and the discussion thereafter, and the SU(3) group lattice is described in Appendix A. 
a $Z_{3}$ symmetry makes a connection between the sectors. More details for the SU(3) group structure can be found in Appendix A.

\section{TUNNELING OPERATORS AND MODELS}

At the intermediate fixed point, there is neither charge nor thermal equilibration between the different modes, implying a nonzero thermal conductance. We now want to describe equilibration between the charge modes (which also includes the creation of neutral excitations) but still assume that the size of the system is shorter than the inelastic scattering length, at which the edge thermal conductance decays towards zero (see Appendix D for a justification of this assumption by a $\mathrm{RG}$ analysis). To this end, we consider quasiparticle tunneling ("charge equilibration") between the outermost mode and the three inner modes, expressed in terms of the most relevant operators:

$$
H_{\text {ch-eq }}=\int d x\left[\left(t_{u} \psi_{u}^{\dagger}+t_{d} \psi_{d}^{\dagger}+t_{s} \psi_{s}^{\dagger}\right) e^{-i \phi_{c} / \sqrt{3}} e^{i \phi_{1 / 3}}+\text { H.c. }\right] .
$$

Note that $H_{\text {ch-eq }}$ breaks the SU(3) symmetry. A quasiparticle $e^{i \phi_{1 / 3}}$ is annihilated, creating a chargeon $e^{-i \phi_{c} / \sqrt{3}}$ (the charge sector of a quasiparticle excitation carrying a charge $e / 3$ formed in the inner modes) and a neutralon. Conversely, while a quasiparticle is created, a chargeon is annihilated together with the creation of an antineutralon (the antiparticle of the neutralon). The microscopic origin of Eq. (10) is presented in Appendix B.

We next recover the $\mathrm{SU}(3)$ symmetry through disorder averaging. The tunneling amplitudes $t_{j=u, d, s}$ are random with white noise correlation function $\left\langle t_{j_{1}}(x) t_{j_{2}}^{*}\left(x^{\prime}\right)\right\rangle_{\text {dis }}=$ $W_{\text {ch-eq }} \delta_{j_{1} j_{2}} \delta\left(x-x^{\prime}\right)$. Note that the correlation function is invariant under $\mathrm{SU}(3)$ rotations owing to the random mixing of the neutral fields; a derivation can be found in Appendix C. The renormalization group scaling of the disorder variance $W_{\text {ch-eq }}$ gives rise to the elastic scattering length $\ell_{\text {ch-eq, } 0} \sim$ $1 / W_{\text {ch-eq }}^{3 / 5}$ (see details in Appendix D). After performing the disorder averaging, an effective action on the Keldysh contour $K$ reads

$$
\begin{aligned}
S_{\text {ch-eq,eff }}= & i W_{\text {ch-eq }} \sum_{j} \int d x \int_{K} d t d t^{\prime} e^{i \phi_{1 / 3}(x, t)} e^{-i \phi_{c}(x, t) / \sqrt{3}} \\
& \times \psi_{j}^{\dagger}(x, t) \psi_{j}\left(x, t^{\prime}\right) e^{i \phi_{c}\left(x, t^{\prime}\right) / \sqrt{3}} e^{-i \phi_{1 / 3}\left(x, t^{\prime}\right)} .
\end{aligned}
$$

This action is invariant under the SU(3) transformation $\psi_{j}(x) \rightarrow U(x) \psi_{j}(x)$, unlike the Hamiltonian equation (10); the SU(3) symmetry of the Hamiltonian equation (7) is thus restored in a statistical sense.

We next discuss a discrete transformation $T_{3}$ defined via $T_{3}^{\dagger}\left(\phi_{c} / \sqrt{3}\right) T_{3}=\left(\phi_{c} / \sqrt{3}-2 \pi / 3\right), \quad T_{3}^{\dagger}\left(\phi_{n_{2}} / \sqrt{2}\right) T_{3}=$ $\left(\phi_{n_{2}} / \sqrt{2}-2 \pi / 3\right)$, and $T_{3}^{\dagger} \phi_{n_{1}} T_{3}=\phi_{n_{1}}$. In the original basis, $T_{3}^{\dagger} \phi_{4} T_{3}=\phi_{4}-2 \pi / 3$ creates a kink, associated with the annihilation of a charge $e / 3$ quasiparticle in $\phi_{4}$ [cf. Fig. 1(a)]. Such properties of $T_{3}$ suggest the explicit form of $T_{3}=e^{i \int_{-\infty}^{\infty} d x \partial_{x} \phi_{4}(x)}$. In the basis of neutralons,

$$
T_{3}^{\dagger} \psi_{j} T_{3}=\psi_{j} e^{2 \pi i / 3}, \quad T_{3}^{\dagger} \psi_{j} T_{3}=\psi_{j} e^{-2 \pi i / 3}
$$

for $j=u, d, s$, reflecting the $Z_{3}$ nature of $T_{3}$. We find that $T_{3}$ is a symmetry of $H_{0}+H_{\text {ch-eq }}$ [Eqs. (7) and (10)]; here, a quasiparticle is not allowed to tunnel into $\phi_{4}$.

Electron tunneling among the original chiral modes is strong near the intermediate fixed point. Provided that the distance between those chiral modes is not too large, the $v=0$ strip [Fig. 1(a)] will be smeared, and quasiparticle tunneling to and from $\phi_{4}$ will be facilitated, reaching the reconstructed edge structure depicted in Fig. 1(b). This, in terms of the renormalized modes, will give rise to the following Hamiltonian in the neutral sector (assumed to be a small perturbation):

$$
H_{n \bar{n}}=\int d x\left(v_{u} \psi_{\bar{d}}^{\dagger} \psi_{s}+v_{d} \psi_{\bar{s}}^{\dagger} \psi_{u}+v_{s} \psi_{\bar{u}}^{\dagger} \psi_{d}+\text { H.c. }\right) \text {. }
$$

This Hamiltonian describes neutralon-antineutralon mixing. For the microscopic origin of Eq. (13), see Appendix B. In view of Eq. (12), $H_{n \bar{n}}$ breaks the $Z_{3}$ symmetry. Here, the annihilation operator of antineutralons is defined as $\psi_{\bar{j}=\bar{u}, \bar{d}, \bar{s}}=$ $\psi_{j}^{\dagger}$. We note that Eq. (13) is the neutralon analog of a BCS Hamiltonian. The Hamiltonian, Eq. (13), also breaks the SU(3) symmetry. Note that the tunneling amplitudes $v_{j=u, d, s}$ are random with a white noise correlator $\left\langle v_{j_{1}}(x) v_{j_{2}}^{*}\left(x^{\prime}\right)\right\rangle_{\text {dis }}=$ $W_{n \bar{n}} \delta\left(x-x^{\prime}\right) \delta_{j_{1} j_{2}}$. Note that the tunneling amplitudes $v_{j=u, d, s}$ have the same disorder correlation $W_{n \bar{n}}$ due to the random rotation of the neutral fields by disorder. This allows us to restore the SU(3) symmetry by performing disorder averaging. The characteristic elastic length scale $\ell_{n \bar{n}, 0}$ for this process scales as $\ell_{n \bar{n}, 0} \sim 1 / W_{n \bar{n}}^{3 / 7}$

Unless the $Z_{3}$ symmetry is broken, the fundamental representation furnished by neutralons is completely disconnected from its conjugate representation furnished by antineutralons within fixed charge sectors. To see this, we consider a correlator $M_{j \rightarrow \bar{j}^{\prime}} \equiv\left\langle 0\left|\psi_{\bar{j}^{\prime}}\left(x_{f}, t_{f}\right) \psi_{j}^{\dagger}\left(x_{i}, t_{i}\right)\right| 0\right\rangle$ between neutralon $j=$ $u, d, s$ at position $x_{i}$ and time $t_{i}$ and antineutralon $\bar{j}^{\prime}=\bar{u}, \bar{d}, \bar{s}$ at position $x_{f}$ and time $t_{f}$. Here, $\psi_{\bar{j}^{\prime}}\left(x_{f}, t_{f}\right)$ and $\psi_{j}^{\dagger}\left(x_{i}, t_{i}\right)$ are operators in the Heisenberg picture. We assume that $Z_{3}$ is a symmetry of the system: $\left[H_{0}+H_{\text {ch-eq }}, T_{3}\right]=0$. Then, the vacuum state $|0\rangle$ must be an eigenstate of $T_{3}, T_{3}|0\rangle=t_{0}|0\rangle$. Employing the symmetry condition $\left[T_{3}, H_{0}+H_{\text {ch-eq }}\right]=0$ and Eq. (12), $M_{j \rightarrow \bar{j}^{\prime}}$ is shown to be zero since

$$
\begin{aligned}
M_{j \rightarrow \bar{j}^{\prime}}= & \left\langle 0\left|U^{\dagger}\left(t_{f}\right) \psi_{\bar{j}^{\prime}}\left(x_{f}\right) U\left(t_{f}-t_{i}\right) \psi_{j}^{\dagger}\left(x_{i}\right) U\left(t_{i}\right)\right| 0\right\rangle \\
= & \langle 0| T_{3} T_{3}^{\dagger} U^{\dagger}\left(t_{f}\right) T_{3} T_{3}^{\dagger} \psi_{\bar{j}^{\prime}}\left(x_{f}\right) T_{3} T_{3}^{\dagger} U\left(t_{f}-t_{i}\right) T_{3} T_{3}^{\dagger} \\
& \times \psi_{j}^{\dagger}\left(x_{i}\right) T_{3} T_{3}^{\dagger} U\left(t_{i}\right)|0\rangle \\
= & e^{-4 \pi i / 3} M_{j \rightarrow \bar{j}^{\prime}}
\end{aligned}
$$

Here, the operator $T_{3}^{\dagger}$ was moved from left to right using the fact that it commutes with the time evolution operator $U(t)=e^{-i\left(H_{0}+H_{\text {ch-eq }}\right) t}$, and $\left|t_{0}\right|^{2}=1$ is used. Since Eq. (14) can only be satisfied for $M_{j \rightarrow \bar{j}^{\prime}}=0$, we conclude that in order to allow mixing between neutralons and antineutralons, the $Z_{3}$ symmetry should be broken. In the next section, we will explicitly show that the conductance plateau of $e^{2} /(3 h)$ is achieved only if this is indeed the case.

We next consider the two-terminal setup depicted in Fig. 2; the QPC is set such that the outermost mode is fully transmitted while the inner modes are fully reflected. Such a QPC configuration naively (neglecting possible contributions from 
neutralons) gives rise to the conductance of $e^{2} /(3 h)$ to drain D. After transmission through or reflection from the QPC, the biased charge modes start to equilibrate with the unbiased ones via quasiparticle tunneling [described by Eq. (10)] in the upper right or lower left corner of Fig. 2(a). Such tunneling events generate neutralons in the upper right corner or antineutralons in the lower left corner, which then propagate in a direction opposite to that of the charge modes through the QPC region with size $L_{\mathrm{QPC}}$ and finally decay in the lower right or upper left corner ("decay region") as depicted in Fig. 2(b). Depending on whether the system exhibits the $Z_{3}$ symmetry or not, we distinguish between two models: Model B includes the terms of Eq. (13) to break the $Z_{3}$, while model A does not. For a clear contrast between the models, $\ell_{n \bar{n}} \ll L_{\mathrm{QPC}}$ is assumed for model B to achieve the complete mixing between neutralons and antineutralons. In both models A and B, we assume that the $\mathrm{SU}(3)$ symmetry is statistically conserved, with $\ell_{\text {ch-eq }, 0} \ll L_{\text {arm }}$.

\section{TRANSPORT PROPERTIES: TUNNELING CURRENT AND NONEQUILIBRIUM NOISE}

We now turn our attention to transport properties of the two-terminal setup: dc conductance and nonequilibrium noise. We first demonstrate that the $Z_{3}$ symmetry of model A prevents the formation of a $e^{2} /(3 h)$ conductance plateau. To understand this, it suffices to consider a single impurity in the decay region in the lower or upper edge $r=1 / \mathrm{u}$. The impurity is assumed to be at position $x_{0}$. Let us start with model A. To leading order in $t_{j}$, the tunneling current at $x_{0}$ on the $r=1$, $\mathrm{u}$ edge can be expressed in terms of a local greater (lesser) Green's function $g_{j, r}^{>}(t)=-i\left\langle\psi_{j, r}\left(x_{0}, t\right) \psi_{j, r}^{\dagger}\left(x_{0}, 0\right)\right\rangle$ $\left[g_{j, r}^{<}(t)=-i\left\langle\psi_{j, r}^{\dagger}\left(x_{0}, 0\right) \psi_{j, r}\left(x_{0}, t\right)\right\rangle\right]$ of $j=u, d, s$ type neutralons as

$$
\begin{aligned}
I_{\text {tun }, r}= & \sum_{j=u, d, s} \frac{i e\left|t_{j}\right|^{2}}{3(a h)^{2}} \int_{-\infty}^{\infty} d t\left(\frac{a}{a+i v_{c} t}\right)^{1 / 3} \\
& \times\left(\frac{a}{a+i v_{1 / 3} t}\right)^{1 / 3}\left[g_{j, r}^{>}(t)-g_{j, r}^{<}(-t)\right] .
\end{aligned}
$$

Here, $a$ is a short-distance cutoff, and $v_{1 / 3}$ and $v_{c}$ are the velocity of the outermost mode and the inner charge mode, respectively. Information about the nonequilibrium state of neutralons is encoded in the local greater and lesser Green's function [40-45] via the decomposition

$$
g_{j, r}^{>}(t)=g_{0}(t) g_{r}^{\mathrm{neq}}(t), \quad g_{j, r}^{<}(t)=g_{0}(-t) g_{r}^{\mathrm{neq}}(t) .
$$

Here, $g_{0}(t)=-i\left[a /\left(a+i v_{n} t\right)\right]^{2 / 3}$ describes quantum correlations of neutralons, while $g_{r}^{\text {neq }}(t)$ represents classical nonequilibrium aspects of neutralons, as

$$
g_{r, \text { model A }}^{\text {neq }}(t)=\left[\frac{1}{2}+\frac{1}{2} e^{2 \pi i r \operatorname{sgn}(t) / 3}\right]^{N},
$$

with $N=e V|t| / \hbar$. We briefly sketch the derivation of Eq. (16). In the limit of full charge equilibration, quasiparticles emanating from source $S$ and then being transmitted through the QPC towards drain D reach the charge mode with probability $1 / 2$. This is accompanied by the creation of neutralons with probability $1 / 2$ [cf. Eq. (17)]. Each of these neutralons arrives at $x_{0}$, described by a kink in the bosonic fields $\phi_{n_{1}}\left(x_{0}\right)$ and $\phi_{n_{2}}\left(x_{0}\right)$, and thus gives rise to a phase shift $2 \pi \operatorname{sgn}(t) / 3$ of the operator $\psi_{j}\left(x_{0}, t\right) \psi_{j}^{\dagger}\left(x_{0}, 0\right)$ : Following the arrival of a $j^{\prime}=u, d, s$ neutralon at $x_{0}$ and time $t^{\prime}, \psi_{j}\left(x_{0}, t\right) \psi_{j}^{\dagger}\left(x_{0}, 0\right)$ acquires a phase shift of $2 \pi \operatorname{sgn}(t) / 3$. This relies on

$$
\begin{aligned}
& \psi_{j^{\prime}}\left(x_{0}, t^{\prime}\right)\left[\psi_{j}\left(x_{0}, t\right) \psi_{j}^{\dagger}\left(x_{0}, 0\right)\right] \psi_{j^{\prime}}^{\dagger}\left(x_{0}, t^{\prime}\right) \\
& \quad=\left[\psi_{j}\left(x_{0}, t\right) \psi_{j}^{\dagger}\left(x_{0}, 0\right)\right] e^{\pi i\left[\operatorname{sgn}\left(t-t^{\prime}\right)-\operatorname{sgn}\left(0-t^{\prime}\right)\right] / 3},
\end{aligned}
$$

provided $\min (0, t)<t^{\prime}<\max (0, t)$. For each arriving neutralon, the phase factor on the right-hand side of Eq. (18) appears in one of the $N$ factors of Eq. (17). We note that the phase factor reflects the anyonic statistics of neutralons and is identical for all flavors $j^{\prime}=u, d, s$. When no neutral excitation is generated (with a probability $1 / 2$ ) during the equilibration process, $\psi_{j}\left(x_{0}, t\right) \psi_{j}^{\dagger}\left(x_{0}, 0\right)$ does not accumulate a phase factor, leading to the first term in brackets in Eq. (17). Inserting Eqs. (16) and (17) into Eq. (15), we obtain the tunneling current as

$$
I_{\mathrm{tun}, r}=\sum_{j=u, d, s} \frac{2 r c e\left|t_{j}\right|^{2}}{3 \hbar \Gamma\left(\frac{4}{3}\right)}\left(\frac{e V}{a^{2} h^{4} v_{n}^{2} v_{c} v_{1 / 3}}\right)^{\frac{1}{3}},
$$

where $\quad c=\sin \left(\tan ^{-1}\{\pi /[3 \ln (2)]\} / 3\right)\left\{(\pi / 3)^{2}+[\ln (2)]^{2}\right\}$ and $\Gamma(x)$ is the gamma function. The finite value of the tunneling current causes a deviation of the conductance from $e^{2} /(3 h)$. If several impurities are taken into account and all the neutral excitations eventually decay $\left(\ell_{\text {ch-eq }, 0} \ll L_{\text {arm }}\right)$, it can be self-consistently shown that the conductance between source and drain is zero up to an exponential correction $\sim \exp \left(-L_{\text {arm }} / \ell_{\text {ch-eq, } 0}\right)$ (see Appendix E for a derivation).

In the framework of model $\mathrm{B}$, the full mixing between neutralons and antineutralons in the QPC region $\left(\ell_{n \bar{n}, 0} \ll L_{\mathrm{QPC}}\right)$ causes both types of particles to arrive with the same probability at $x_{0}$. As the phase shifts of neutralons and antineutralons are the complex conjugate of each other, the nonequilibrium part of the Green's functions

$$
g_{r, \text { model B }}^{\text {neq }}(t)=\left[\frac{1}{2}+\frac{1}{4} e^{2 \pi i \operatorname{sgn}(t) / 3}+\frac{1}{4} e^{-2 \pi i \operatorname{sgn}(t) / 3}\right]^{N} .
$$

is real and leads to a vanishing tunneling current when inserted into Eqs. (15) and (16), causing the conductance quantization of $e^{2} /(3 h)$. The incomplete mixing of neutralons with antineutralons leads to an exponential correction $\propto \exp \left(-L_{\mathrm{QPC}} / \ell_{n \bar{n}, 0}\right)$ of the quantized value.

We now quantify the zero-frequency nonequilibrium noise measured at the drain for model $\mathrm{B}$. Using the nonequilibrium bosonization technique [40-45], we compute generating functions $g_{n_{1}}=\left\langle e^{i \lambda_{1} \phi_{n_{1}}(x, t)} e^{-i \lambda_{1} \phi_{n_{1}}\left(x^{\prime}, t^{\prime}\right)}\right\rangle$ and $g_{n_{2}}=$ $\left\langle e^{i \lambda_{2} \phi_{n_{2}}(x, t)} e^{-i \lambda_{2} \phi_{n_{2}}\left(x^{\prime}, t^{\prime}\right)}\right\rangle$ as

$$
\begin{gathered}
g_{n_{1}}=\left(\frac{a}{a+i v_{n} \tau}\right)^{\lambda_{1}^{2}}\left[\frac{2}{3}+\frac{\cos \left(\pi \sqrt{2} \lambda_{1}\right)}{3}\right]^{M}, \\
g_{n_{2}}=\left(\frac{a}{a+i v_{n} \tau}\right)^{\frac{\lambda_{2}^{2}}{3}}\left[\frac{1}{2}+\frac{\cos \left(\frac{\sqrt{2} \pi \lambda_{2}}{3}\right)}{3}+\frac{\cos \left(\frac{2 \sqrt{2} \pi \lambda_{2}}{3}\right)}{6}\right]^{M},
\end{gathered}
$$

valid in the long-time limit $M \equiv e V|\tau| / h \gg 1$, with $\tau \equiv$ $\left(t-t^{\prime}\right)+\left(x-x^{\prime}\right) / v_{n}$. To derive Eqs. (21) and (22), we have used that up and down neutralons create a kink of height 
$\pm \sqrt{2} \pi$ in $\phi_{n 1}$, while the strange neutralons leave $\phi_{n 1}$ invariant. Likewise, up and down neutralons create a kink of height $+\sqrt{2} \pi / 3$ in $\phi_{n_{2}}$, while strange neutralons create a kink of height $-2 \sqrt{2} \pi / 3$.

By taking second derivatives, we obtain the correlation functions $C_{n_{i}}=\left\langle\phi_{n_{i}}(x, t) \phi_{n_{i}}\left(x^{\prime}, t^{\prime}\right)\right\rangle-\left\langle\phi_{n_{i}}^{2}(x, t)\right\rangle(i=1,2$ labels the two neutral modes) as

$$
\begin{aligned}
& C_{n_{1}}(\tau)=\ln \left(\frac{a}{a+i v_{n} \tau}\right)-\frac{e V|\tau| \pi^{2}}{3 h}, \\
& C_{n_{2}}(\tau)=\frac{1}{3} \ln \left(\frac{a}{a+i v_{n} \tau}\right)-\frac{e V|\tau| \pi^{2}}{9 h} .
\end{aligned}
$$

The noise in the neutral currents $I_{n_{1}}(x, t)=v_{n} \partial_{x} \phi_{n_{1}} /(\sqrt{2} \pi)$ and $I_{n_{2}}(x, t)=3 v_{n} \partial_{x} \phi_{n_{2}} /(\sqrt{2} \pi)$ evaluated in the decay region of edge $r=\mathrm{u} / 1$ is defined as $S_{n_{i=1,2}, r} \equiv$ $2 \int d t\left\langle I_{n_{1}}\left(x_{r}, t\right) I_{n_{1}}\left(x_{r}, 0\right)\right\rangle$. Employing Eq. (23), we obtain

$$
\begin{aligned}
S_{n_{1}, \mathrm{u} / 1} & =\left.\frac{v_{n}^{2}}{\pi^{2}} \int_{-\infty}^{\infty} d t \partial_{x} \partial_{x^{\prime}} C_{n_{1}}(\tau)\right|_{x=x^{\prime}, t^{\prime}=0} \\
& =-\frac{1}{\pi^{2}} \int_{-\infty}^{\infty} \partial_{\tau}^{2} C_{n_{1}}(\tau) d \tau=\frac{2 e V}{3 h}, \\
S_{n_{2}, \mathrm{u} / 1} & =\frac{2 e V}{h} .
\end{aligned}
$$

We emphasize that even though the second term in Eq. (23) only holds for the large $|\tau|$ limit, Eq. (24) is valid because it only depends on the values of $\partial_{\tau} C_{n_{1}}$ and $\partial_{\tau} C_{n_{2}}$ at $\tau \rightarrow \pm \infty$; it is insensitive to details of $\partial_{\tau} C_{n_{1}}$ and $\partial_{\tau} C_{n_{2}}$ at small $\tau$. Due to the decay of all neutral excitations, the electrical noise measured at drain D can be expressed as

$$
S_{\mathrm{D}}=\frac{e^{2}}{36}\left(3 S_{n_{1}, \mathrm{u}}+S_{n_{2}, \mathrm{u}}+3 S_{n_{1}, 1}+S_{n_{2}, 1}\right) .
$$

We refer the reader to Appendix F for a derivation of Eq. (25). Employing Eqs. (24) and (25), we obtain the Fano factor characterizing the strength of noise at $\mathrm{D}$ as

$$
F \equiv S_{\mathrm{D}} /\left[2 I_{\mathrm{imp}} T(1-T)\right]=2 e / 3 .
$$

Here, we used that the current impinging on the QPC is given by $I_{\mathrm{imp}}=2 e^{2} V /(3 h)$ and that the transmission probability through the QPC is $T=1 / 2$.

Experimental manifestations (conductance and dc noise) of possible symmetry scenarios beyond those of models A and $\mathrm{B}$ are summarized in Table I. For example, when $Z_{3}$ is conserved and the $\mathrm{SU}(3)$ symmetry is exact [intermediate $\mathrm{SU}(3)$-symmetric fixed point], the conductance is quantized at $e^{2} /(3 h)$ with vanishing noise (there is no tunneling between the charge modes as $\left.\ell_{\text {ch-eq, } 0} \rightarrow \infty\right)$. In yet another scenario, $\ell_{\text {ch-eq, } 0}$ becomes finite, but still $\ell_{\text {ch-eq, } 0} \ll L_{\text {arm }}$. Then, some quasiparticles emanating from the source are reflected via the two-step mechanism of neutralon creation followed by the decay of these neutralons, leading to a reduction of the quantized conductance. While mesoscopic fluctuations of the conductance $[30,31]$ occur when $\ell_{\text {ch-eq, } 0} \gg L_{\text {arm }}$, both conductance and noise become zero when $\ell_{\text {ch-eq, } 0} \ll L_{\text {arm }}$. When the $Z_{3}$ symmetry is broken (as $\ell_{n \bar{n}, 0} \ll L_{\mathrm{QPC}}$ ), on the other hand, the conductance is always quantized as $e^{2} /(3 h)$. The Fano factor, however, deviates from the nonuniversal value to $2 e / 3$ as $\ell_{\text {ch-eq, } 0}$ decreases. This nonuniversal Fano factor can be attributed to a partial decay of neutral excitations, producing a smaller noise as compared with the case of full decay. The crossover of the transport properties between $\ell_{\text {ch-eq, } 0} \gg L_{\text {arm }}$ and $\ell_{\text {ch-eq, } 0} \ll L_{\text {arm }}$ might be experimentally tuned by varying either $L_{\text {arm }}$ directly or the slope of the edge confining potential to effectively control $\ell_{\text {ch-eq, } 0}$.

\section{SUMMARY}

For general hole-conjugate quantum Hall states at filling factor $v=-n /(n p+1)$, with negative even integer $p$ and positive integer $n$, the relevant symmetries to be considered are a $\mathrm{SU}(n+1)$ symmetry in the neutralon sector and a $Z_{n+1}$ symmetry connecting neutralons to antineutralons. Similarly to the $2 / 3$ case, we expect different symmetry scenarios, including a quantized noise on a conductance plateau for statistically preserved $\mathrm{SU}(n+1)$ and broken $Z_{n+1}$.

To summarize, we have investigated the roles of two symmetries, a continuous $\mathrm{SU}(3)$ symmetry and a discrete $Z_{3}$ symmetry, in influencing the two-terminal conductance and the dc noise of a quantum Hall strip at filling factor $v=2 / 3$ with a single QPC. While recent measurements $[11,13]$ with a Fano factor $2 e / 3$ on the conductance plateau of $e^{2} /(3 h)$ are explained relying on a broken $Z_{3}$ symmetry, other symmetry scenarios (summarized in Table I) can be realized in future experiments.

\section{ACKNOWLEDGMENTS}

We thank Amir Rosenblatt, Rajarshi Bhattacharyya, Ron Sabo, Itamar Gurman, Christian Spånslätt, and Moty Heiblum for helpful discussions. We are indebted to Alexander Mirlin for very useful comments on the manuscript. B.R. acknowledges support from the Rosi and Max Varon Visiting Professorship at the Weizmann Institute of Science, B.R. and Y.G. acknowledge support from DFG Grant No. RO 2247/11-1. Y.G. further acknowledges support from CRC 183 (Project C01), the Minerva Foundation, and DFG Grant No. MI 658/10-1. J.P. acknowledges support by the Koshland Foundation and funding by the Deutsche Forschungsgemeinschaft (DFG, German Research Foundation) - Projektnummer 277101999 - TRR 183 (project A01).

\section{APPENDIX A: SU(3) GROUP STRUCTURE}

In this Appendix, we consider the intermediate fixed point vis-à-vis the $S U(3)$ group structure. As seen in Eq. (1), the intermediate $\mathrm{SU}(3)$-symmetric fixed point is given by

$$
\begin{aligned}
S_{n}=\int d x d t & {\left[\frac{1}{4 \pi}\left[\partial_{x} \phi_{n_{1}}\left(\partial_{t}-v_{n} \partial_{x}\right) \phi_{n_{1}}+3 \partial_{x} \phi_{n_{2}}\left(\partial_{t}-v_{n} \partial_{x}\right) \phi_{n_{2}}\right]\right.} \\
& \left.-\frac{1}{2 \pi a}\left(\xi_{1}(x) e^{i \sqrt{2} \phi_{n_{1}}}+\xi_{2}(x) e^{-i \phi_{n_{1}} / \sqrt{2}} e^{3 i \phi_{n_{2}} / \sqrt{2}}+\xi_{3}(x) e^{i \phi_{n_{1}} / \sqrt{2}} e^{3 i \phi_{n_{2}} / \sqrt{2}}+\text { H.c. }\right)\right] .
\end{aligned}
$$




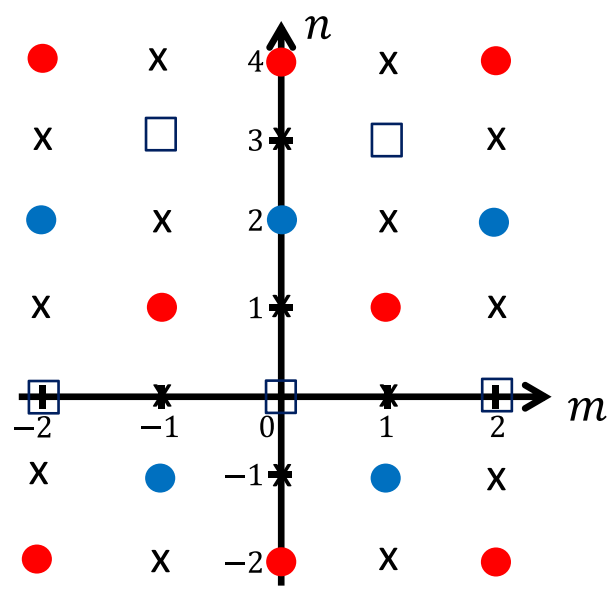

FIG. 4. A lattice for SU(3) group representations with allowed values of $m$ and $n$ for states of $e^{i m \phi_{n_{1}} / \sqrt{2}} e^{i n \phi_{n_{2}} / \sqrt{2}}|0\rangle$. Here, $|0\rangle$ is the vacuum state. The points denoted as $\times$ are not allowed. The states are divided into three sublattices: the neutralon sector (denoted as red dots), the antineutralon sector (denoted as blue dots), and the vacuum sector (denoted as rectangles). Points within each sublattice are connected via the electron operators between the inner modes [written in the second line of Eq. (1)], while a sublattice is completely decoupled from the other sublattices due to Eq. (1). The presence of three different sectors manifests the $Z_{3}$ subgroup of the SU(3) group.

The second line originates from electron tunneling between the inner modes; $\xi_{i=1,2,3}$ are random variables. It is worthwhile to note the group structure of Eq. (A1). A lattice for $\mathrm{SU}(3)$ group representations with allowed values of $m$ and $n$ for states of $e^{i m \phi_{n_{1}} / \sqrt{2}} e^{i n \phi_{n_{2}} / \sqrt{2}}|0\rangle$ is displayed in Fig. 4. Here, $|0\rangle$ is the vacuum state. The points denoted as $\times$ are not allowed. The states are divided into three sublattices: the neutralon sector (denoted as red dots), the antineutralon sector (denoted as blue dots), and the vacuum sector (denoted as rectangles). Points within each sublattice are connected via the electron operators between the inner modes [written in the second line of Eq. (A1)], while a sublattice is completely decoupled from the other sublattices by the electron tunneling. The presence of the three disconnected sectors manifests the $Z_{3}$ subgroup of the $\mathrm{SU}(3)$ group. Importantly, it should be noted that the neutralon sector is completely disconnected from the antineutralon sectors by the SU(3) symmetry. For the connection between the neutralon and the antineutralon sectors, the discrete symmetry $T_{3}$ [defined in Eq. (12)] should be broken.

Including the action for an auxiliary field $\chi$ (see Ref. [28] for a similar procedure) and performing refermionization in terms of a three-component fermion field $\Psi(x)=$ $e^{i \chi / \sqrt{3}}\left[e^{-i\left(\phi_{n_{1}}+\phi_{n_{2}}\right) / \sqrt{2}}, e^{i\left(\phi_{n_{1}}-\phi_{n_{2}}\right) / \sqrt{2}}, e^{i \sqrt{2} \phi_{n_{2}}}\right]^{T} / \sqrt{2 \pi a}=e^{i \chi / \sqrt{3}}$ $\left[\psi_{u}, \psi_{d}, \psi_{s}\right]^{T} / \sqrt{2 \pi a}$, Eq. (A1) reads

$$
\begin{aligned}
& S_{n}=\int d x d t\left\{i \Psi^{\dagger}\left(-\partial_{t}+v_{n} \partial_{x}\right) \Psi\right. \\
&-\left[\xi_{1} \Psi^{\dagger}\left(\frac{\lambda_{1}+i \lambda_{2}}{2}\right) \Psi+\xi_{2} \Psi^{\dagger}\left(\frac{\lambda_{6}+i \lambda_{7}}{2}\right) \Psi\right. \\
&\left.\left.+\xi_{3} \Psi^{\dagger}\left(\frac{\lambda_{4}+i \lambda_{5}}{2}\right) \Psi+\text { H.c. }\right]\right\} .
\end{aligned}
$$

$\lambda_{1,2,4,5,6,7}$ are nondiagonal generators of the $\mathrm{SU}(3)$ group, given by

$$
\begin{aligned}
& \lambda_{1}=\left(\begin{array}{lll}
0 & 1 & 0 \\
1 & 0 & 0 \\
0 & 0 & 0
\end{array}\right), \quad \lambda_{2}=\left(\begin{array}{ccc}
0 & -i & 0 \\
i & 0 & 0 \\
0 & 0 & 0
\end{array}\right), \\
& \lambda_{4}=\left(\begin{array}{lll}
0 & 0 & 1 \\
0 & 0 & 0 \\
1 & 0 & 0
\end{array}\right), \\
& \lambda_{5}=\left(\begin{array}{ccc}
0 & 0 & -i \\
0 & 0 & 0 \\
i & 0 & 0
\end{array}\right), \quad \lambda_{6}=\left(\begin{array}{ccc}
0 & 0 & 0 \\
0 & 0 & 1 \\
0 & 1 & 0
\end{array}\right), \\
& \lambda_{7}=\left(\begin{array}{ccc}
0 & 0 & 0 \\
0 & 0 & -i \\
0 & i & 0
\end{array}\right) .
\end{aligned}
$$

The diagonal generators $\lambda_{3}$ and $\lambda_{8}$ are associated with the density of the neutral modes, $\partial_{x} \phi_{n_{1}}$ and $\partial_{x} \phi_{n_{2}}$, and are explicitly written in Eq. (9). The random terms are completely eliminated via performing a SU(3) gauge transformation of $\tilde{\Psi}(x)=U(x) \Psi(x)$; the action becomes diagonal as $S_{n}=i \int d x d t \tilde{\Psi}^{\dagger}\left(-\partial_{t}+v_{n} \partial_{x}\right) \tilde{\Psi}$. The position-dependent $\mathrm{SU}(3)$ matrix $U(x)$ is given in the main text [Eq. (4)].

\section{APPENDIX B: TUNNELING OPERATORS}

In this Appendix, we derive the form of quasiparticle tunneling operators in terms of neutralons.

We first consider quasiparticle tunneling between the inner modes and the outermost mode in the vicinity of the intermediate fixed point, given by

$$
\begin{aligned}
H_{\text {ch-eq }}= & \int d x\left[t_{u, 0} e^{i\left(\phi_{1 / 3}+\phi_{2}\right)}+t_{d, 0} e^{i\left(\phi_{1 / 3}-2 \phi_{2}-\phi_{3}\right)}\right. \\
& \left.+t_{s, 0} e^{i\left(\phi_{1 / 3}-2 \phi_{2}-2 \phi_{3}-3 \phi_{4}\right)}+\text { H.c. }\right] \\
= & \int d x\left[\left(t_{u} \psi_{u}^{\dagger}+t_{d} \psi_{d}^{\dagger}\right.\right. \\
& \left.\left.+t_{s} \psi_{s}^{\dagger}\right) e^{-i \phi_{c} / \sqrt{3}} e^{i \phi_{1 / 3}}+\text { H.c. }\right] .
\end{aligned}
$$

All the tunneling operators have the same scaling dimension of $\delta_{\text {ch-eq }}=2 / 3$. Bare tunneling amplitudes $t_{j, 0}$ are random with white noise correlation $\left\langle t_{j_{1}, 0}(x) t_{j_{2}, 0}^{*}\left(x^{\prime}\right)\right\rangle_{\mathrm{dis}}=W_{\mathrm{eq}, j_{1}}^{0} \delta(x-$ $\left.x^{\prime}\right) \delta_{j_{1} j_{2}}$. In the rotated basis of the $\psi_{j}$, the tunneling amplitudes $t_{j=u, d, s}$ are given by $t_{j}=\sum_{j^{\prime}} U_{j j^{\prime}} t_{j^{\prime}, 0}$.

The other type of quasiparticle tunneling (related to quasiparticle tunneling to the $\phi_{4}$ mode) occurs when the electron tunneling between the inner modes is strong enough to eliminate the regions of the filling factors $v=1$ and $v=0$, eventually reaching the edge structure of Fig. 1(b). The most relevant terms are written as

$$
\begin{aligned}
H_{n \bar{n}}= & \int d x\left(v_{u, 0} e^{i\left(2 \phi_{2}+\phi_{3}+\phi_{4}\right)}+v_{d, 0} e^{-i\left(\phi_{2}-\phi_{4}\right)}\right. \\
& \left.+v_{s, 0} e^{-i\left(\phi_{2}+\phi_{3}+2 \phi_{4}\right)}+\text { H.c. }\right) \\
= & \int d x\left(v_{u} \psi_{\bar{d}}^{\dagger} \psi_{s}+v_{d} \psi_{\bar{s}}^{\dagger} \psi_{u}+v_{s} \psi_{\bar{u}}^{\dagger} \psi_{d}+\text { H.c. }\right) .
\end{aligned}
$$


All the terms have the same scaling dimension of $\delta_{n \bar{n}}=1 / 3$. Bare tunneling amplitudes $v_{j, 0}$ are random with white noise correlation $\left\langle v_{j_{1}, 0}(x) v_{j_{2}, 0}^{*}\left(x^{\prime}\right)\right\rangle_{\mathrm{dis}}=W_{n \bar{n}, j_{1}}^{0} \delta\left(x-x^{\prime}\right) \delta_{j_{1} j_{2}}$.

\section{APPENDIX C: SU(3) SYMMETRY RESTORATION BY DISORDER AVERAGING}

In this Appendix, it is shown that after the self-averaging over the disorder, the effective action for the quasiparticle tunneling becomes invariant under the SU(3) transformation.

To this end, we consider the quasiparticle tunneling [Eq. (B1)] between the outermost mode and the inner ones. The tunneling amplitude $t_{j=u, d, s}$ is random with the noise correlation as

$$
\begin{aligned}
\left\langle t_{j_{1}}(x) t_{j_{2}}^{*}\left(x^{\prime}\right)\right\rangle_{\mathrm{dis}}= & \sum_{j, j^{\prime}}\left\langle U_{j_{1} j}(x) t_{j, 0}(x) U_{j_{2}, j^{\prime}}^{*}\left(x^{\prime}\right) t_{j^{\prime}, 0}\left(x^{\prime}\right)\right\rangle_{\mathrm{dis}} \\
= & \sum_{j, j^{\prime}}\left\langle U_{j_{1} j}(x) U_{j_{2}, j^{\prime}}^{*}\left(x^{\prime}\right)\right\rangle_{\mathrm{dis}} \\
& \times\left\langle t_{j, 0}(x) t_{j^{\prime}, 0}\left(x^{\prime}\right)\right\rangle_{\mathrm{dis}} \\
= & \sum_{j}\left\langle U_{j_{1} j}(x) U_{j_{2}, j}^{*}\left(x^{\prime}\right)\right\rangle_{\mathrm{dis}} W_{\mathrm{eq}, j}^{0} \delta\left(x-x^{\prime}\right)
\end{aligned}
$$

$$
\begin{aligned}
& =U_{0} \delta\left(x-x^{\prime}\right) \delta_{j_{1}, j_{2}} \sum_{j} W_{\mathrm{eq}, j}^{0} \\
& =W_{\text {ch-eq }} \delta\left(x-x^{\prime}\right) \delta_{j_{1}, j_{2}} .
\end{aligned}
$$

Here, the second equality in Eq. (C1) is due to the statistical independence of $U$ and $t_{0}$ with regards to the disorder averaging, and $\sum_{j}\left\langle U_{j_{1} j}(x) U_{j_{2}, j}^{*}\left(x^{\prime}\right)\right\rangle_{\text {dis }}=U_{0} \delta_{j_{1}, j_{2}} \delta\left(x-x^{\prime}\right)$ comes from the randomness of $U$. We defined $W_{\text {ch-eq }} \equiv U_{0} \sum_{j} W_{\text {eq }, j}^{0}$. Equation $(\mathrm{C} 1)$ shows that the variance of the tunneling amplitudes in the rotated basis does not depend on the neutralon flavor. This claim is also applicable to the variances of the tunneling amplitudes for $H_{n \bar{n}}$ [Eq. (13)] through a similar procedure.

Employing this claim, we show that after the self-averaging over the disorder, the effective action becomes invariant under the SU(3) transformation. To this end, we consider the corresponding Keldysh action written as

$$
\begin{gathered}
S_{\text {ch-eq }}=-\int d x \int_{K} d t\left[\left(t_{u} \psi_{u}^{\dagger}+t_{d} \psi_{d}^{\dagger}+t_{s} \psi_{s}^{\dagger}\right) e^{-i \phi_{c} / \sqrt{3}} e^{i \phi_{1 / 3}}\right. \\
+ \text { c.c. }] .
\end{gathered}
$$

The disorder averaging of $e^{i S_{\text {cheq }}}$ can be easily performed by

$$
\left\langle e^{i S_{n \bar{n}}}\right\rangle_{\mathrm{dis}}=\frac{\int D t_{j} D t_{j}^{*} D \psi_{j} D \psi_{j}^{\dagger} \exp \left[-\sum_{j} \int d x t_{j}(x) t_{j}^{*}(x) / W_{\text {ch-eq }}+i S_{n \bar{n}}\right]}{\int D t_{j} D t_{j}^{*} e^{-\sum_{j} \int d x t_{j}(x)_{j}^{*}(x) / W_{\text {ch-eq }}}} .
$$

The integration of $\left\langle e^{i S_{n \bar{n}}}\right\rangle_{\text {dis }} \equiv \int D \psi_{j} D \psi_{j}^{\dagger} e^{i S_{\text {ch-eq,eff }}}$ over the random amplitudes leads to the effective action equation (11). This effective action is invariant under the $\mathrm{SU}(3)$ transformation $\psi_{j}(x) \rightarrow \sum_{j^{\prime}} U_{j j^{\prime}}(x) \psi_{j^{\prime}}(x)$ unlike the original

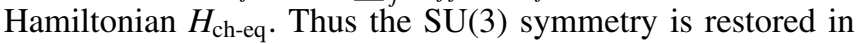
the statistical sense.

\section{APPENDIX D: LENGTH SCALES}

Here, we describe the relevant length scales and discuss their scaling with an external voltage. We analyze it employing the RG method of Ref. [31]. The schematic dependence of the relevant length scales on the voltage is depicted in Fig. 5. We focus on the dependence on the voltage, but the voltage $V$ is perfectly interchangeable with the temperature $T$ when $T \gg V$.

We first summarize all length scales considered in this paper:

(i) $L_{\text {arm }}$ is the length of the arms between the contacts and the QPC.

(ii) $L_{\mathrm{QPC}}$ is the size of the QPC.

(iii) $\ell_{0}$ is the elastic scattering length beyond which disorder-induced electron tunneling mixes the inner modes. When $L_{\text {arm }}$ exceeds $\ell_{0}$, disorder becomes relevant, and the system is driven to the intermediate fixed point [20]. The intermediate fixed point is called the Wang-Meir-Gefen (WMG) fixed point in this Appendix.

(iv) $\ell_{\text {ch-eq }, 0}$ is the elastic scattering length beyond which disorder-induced quasiparticle tunneling mixes the inner modes with the outermost mode. (v) $\ell_{n \bar{n}, 0}$ is the elastic scattering length over which neutralons are mixed with antineutralons.

(vi) $\ell_{\mathrm{ch} \text {-eq }}$ is the inelastic scattering length (the red curve in Fig. 5) over which the charge modes are equilibrated.

(vii) $\ell_{n \bar{n}}$ is the inelastic scattering length over which neutralons are equilibrated with antineutralons.

(viii) $\ell$ is the inelastic scattering length (the yellow curve in Fig. 5) over which the inner three modes equilibrate.

(ix) $\ell_{\text {in }}$ is the coherence length (the thick black curve in Fig. 5) at which the modes lose the coherence. It takes the smaller value between $\ell$ and $\ell_{\text {ch-eq. }}$. This can be, in principle, calculated from the analysis of the Boltzmann kinetics.

(x) $L_{V}$ is the voltage length $\sim 1 / V$ (the thin black line in Fig. 5) operating as an infrared cutoff.

We assume that the electron tunneling operators [Eq. (1)] within the inner modes drive the system to the WMG intermediate fixed point [20]; the length scales $L_{\text {arm }}$ and $L_{\mathrm{QPC}}$ are much larger than $\ell_{0}$, over which the inner modes are strongly mixed by disorder. In the vicinity of the WMG fixed point, we consider quasiparticle tunneling $H_{\mathrm{ch}-\mathrm{eq}}$ between the outermost mode and the inner modes [Eq. (10)] and neutralonantineutralon mixing term $H_{n \bar{n}}$ [Eq. (13)]. Below, we will find the scaling of the length scales associated with $H_{\text {ch-eq }}$ and $H_{n \bar{n}}$ using the RG method. $\ell_{0}$ acts as the ultraviolet length cutoff of the RG analysis.

We first consider $\ell_{\text {ch-eq, } 0}$, characterizing the elastic scattering between the outermost mode and the inner ones. $\ell_{\text {ch-eq, } 0}$ is determined by the following $\mathrm{RG}$ equation:

$$
\frac{d \tilde{W}_{\text {ch-eq }}}{d \ln L}=\tilde{W}_{\text {ch-eq }}\left(3-2 \Delta_{\text {ch-eq }}\right) .
$$




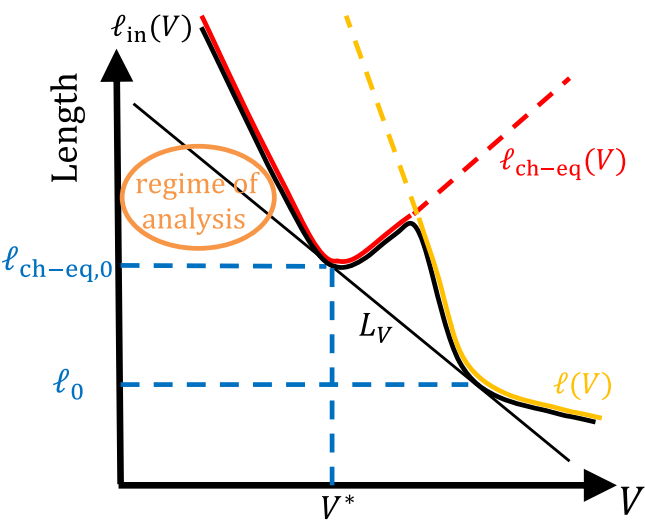

FIG. 5. The schematic dependence of the relevant length scales on the applied voltage $V$ (log-log plot); $\ell_{0}$ and $\ell_{\text {ch-eq }, 0}$ (blue dashed lines) are the voltage-independent elastic lengths. They correspond to elastic scattering within the inner modes and between the inner and outer modes, respectively. The voltage length $L_{V} \propto 1 / V$ (thin black line) acts as an infrared cutoff. $\ell(V)$ (yellow curve) and $\ell_{\text {ch-eq }}(V)$ (red curve) are the voltage-dependent inelastic lengths associated with inelastic scattering within the inner modes and between the inner and outer modes, respectively. Specifically, the scaling of $\ell_{\text {ch-eq }}$ changes from $\ell_{\text {ch-eq }} \propto 1 / V^{2}$ (low voltage) to $\ell_{\text {ch-eq }} \propto V^{2 / 3}$ (high voltage) at the transition voltage $V=V^{*}$. Here, the transition voltage $V^{*}$ is defined as the voltage that satisfies $L_{V}\left(V^{*}\right)=\ell_{\text {ch-eq, } 0}$. The inelastic length $\ell_{\text {in }}(V)$ (at which the modes lose coherence) takes the smaller value between $\ell$ and $\ell_{\text {ch-eq. }}$. Our main focus in this paper is on the regime in which $L_{\text {arm }}$ is much larger than $\ell_{\text {ch-eq }, 0}$ but smaller than $\ell_{\text {ch-eq }}$. The voltage $V$ is perfectly interchangeable with the temperature $T$ when $T \gg V$.

Here, $\tilde{W}_{\text {ch-eq }} \equiv W_{\text {ch-eq }} \ell_{0}^{3} / v_{n}^{2}$ [cf. see the in-line equation above Eq. (11) for $\left.W_{\text {ch-eq }}\right]$ is the dimensionless disorder strength, and $\Delta_{\text {ch-eq }}$ is the scaling dimension of the $H_{\text {ch-eq }}$ [Eq. (10)] and is equal to $2 / 3$ at the fixed point. Assuming trivial densityof-states factors, $\tilde{W}_{\text {ch-eq }}$ can be thought of as the running dimensionless resistance. We now consider the voltage $V$ to be sufficiently small, such that the renormalized $\tilde{W}_{\text {ch-eq }}$ reaches 1 for $L=\ell_{\text {ch-eq, } 0}<L_{V}\left(V<V^{*}\right.$ in Fig. 5). The perturbative RG of Eq. (D1) breaks down when $\tilde{W}_{\text {ch-eq }}$ becomes unity and the corresponding length scale (at which $\tilde{W}_{\text {ch-eq }}$ renormalizes to unity) is $\ell_{\text {ch-eq, } 0}=\ell_{0} /\left(\tilde{W}_{\text {ch-eq }}^{0}\right)^{3 / 5}$. Here, $\tilde{W}_{\text {ch-eq }}^{0}$ is the bare dimensionless disorder strength. Following the same procedure for the $H_{n \bar{n}}$ [Eq. (13)], we also obtain $\ell_{n \bar{n}, 0}=\ell_{0} /\left(\tilde{W}_{n \bar{n}}^{0}\right)^{3 / 7}$. Both $\ell_{n \bar{n}, 0}$ and $\ell_{\text {ch-eq, } 0}$ are elastic scattering lengths and do not depend on energy cutoffs (externally applied voltage $V$ ) of the system.

As $L_{\text {arm }}$ exceeds $\ell_{n \bar{n}, 0}$ and $\ell_{\text {ch-eq, } 0}$, the system is further renormalized away from the WMG fixed point, ultimately arriving at the low-energy fixed point. In the vicinity of the latter fixed point, counterpropagating neutral modes localize each other, leaving a charge mode and a neutral mode [20]. The inelastic length scales (cf. the red curve at $V<V^{*}$ in Fig. 5) near the low-energy fixed point can exceed the elastic charge equilibration length $\ell_{\text {ch-eq, } 0}$ parametrically at sufficiently small voltages [28,31]. By continuity, there exists a regime $\left(\ell_{\text {ch-eq, } 0}<L_{\text {arm }}<\ell_{\text {ch-eq }}\right)$ still governed by the WMG fixed point where our analysis is mostly performed.

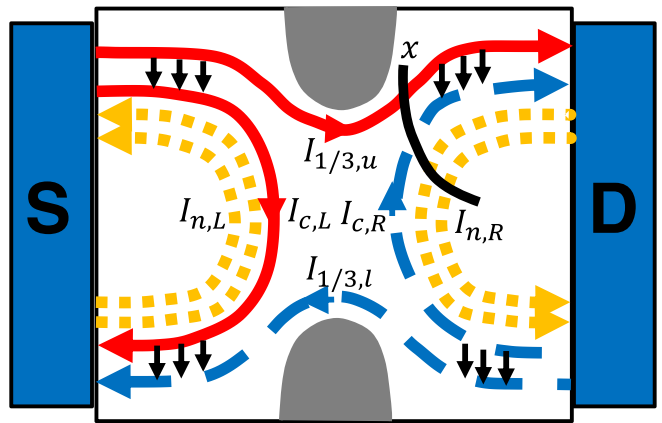

FIG. 6. A two-terminal setup with a quantum point contact (QPC). Source $\mathrm{S}$ is biased by $V_{0}$. The two-terminal conductance is measured between $\mathrm{S}$ and $\mathrm{D}$; the dc noise is measured at drain D. Black arrows denote the direction of tunneling charge currents at each corner.

When the voltage, on the other hand, is sufficiently large such that $\ell_{\text {ch-eq, } 0}$ is larger than $L_{V}\left[V \gg V^{*}\right.$ in Fig. 5], Eq. (10) with Eq. (D1) yields $\ell_{\text {ch-eq }}$. Equation (D1) stops being valid at the infrared cutoff $L_{V}$, leading to

$$
\frac{\tilde{W}_{\text {ch-eq }}\left(L=L_{V}\right)}{\tilde{W}_{\text {ch-eq }}^{0}}=\left(\frac{L_{V}}{\ell_{0}}\right)^{5 / 3} .
$$

Beyond the scale $L_{V}$, the renormalization of the resistance, and hence of $\tilde{W}_{\text {ch-eq }}$, continues classically (i.e., $\tilde{W}_{\text {ch-eq }}$ grows linearly as $L$ increases) [31] and in turn breaks down when $\tilde{W}_{\text {ch-eq }}$ becomes unity, leading to

$$
\frac{\tilde{W}_{\text {ch-eq }}\left(L=L_{V}\right)}{L_{V}}=\frac{\tilde{W}_{\text {ch-eq }}\left(L=\ell_{\text {ch-eq }}\right)}{\ell_{\text {ch-eq }}} \simeq \frac{1}{\ell_{\text {ch-eq }}} .
$$

Here, $\ell_{\text {ch-eq }}$ is defined as the length at which $\tilde{W}_{\text {ch-eq }}$ becomes unity. Employing Eqs. (D2) and (D3), we obtain $\ell_{\text {ch-eq }}=$ $\ell_{0}\left(\ell_{0} / L_{V}\right)^{2 / 3} / \tilde{W}_{\text {ch-eq }}^{0} \propto V^{2 / 3}$ (depicted with the red curve at $V \gg V^{*}$ in Fig. 5). Following the same procedure for the $H_{n \bar{n}}$, we also obtain $\ell_{n \bar{n}}=\ell_{0}\left(\ell_{0} / L_{V}\right)^{4 / 3} / \tilde{W}_{n \bar{n}}^{0} \propto V^{4 / 3}$.

\section{APPENDIX E: THE $Z_{3}$ SYMMETRY AND THE TWO-TERMINAL CONDUCTANCE}

In this Appendix, we show self-consistently that the twoterminal conductance is zero if (i) $Z_{3}$ is present (model A) and (ii) all neutral excitations eventually decay $\left(\ell_{\text {ch-eq, } 0} \ll L_{\text {arm }}\right)$. The quasiparticle tunneling probability between the outermost mode and the inner modes in each corner is defined as $P_{u, R}$, $P_{u, L}, P_{l, R}$, and $P_{l, L}$, respectively. All the electrical currents displayed in Fig. 6 are determined by the following rate equations:

$$
\begin{aligned}
I_{1 / 3, u} & =\frac{e^{2} V_{0}}{3 h}+\frac{e}{3} I_{n, L} P_{u, L}, \\
I_{1 / 3, l} & =\frac{e}{3} I_{n, R} P_{l, R}, \\
I_{c, L} & =\frac{e^{2} V_{0}}{3 h}-\frac{e}{3} I_{n, L} P_{u, L}, \\
I_{c, R} & =-\frac{e}{3} I_{n, R} P_{l, R},
\end{aligned}
$$




$$
\begin{aligned}
& \frac{e}{3} I_{n, L}=\frac{1}{2} P_{l, L}\left(I_{1 / 3, l}-I_{c, L}\right), \\
& \frac{e}{3} I_{n, R}=\frac{1}{2} P_{u, R}\left(I_{1 / 3, u}-I_{c, R}\right) .
\end{aligned}
$$

The conductance measured at $\mathrm{D}$ is calculated as

$$
\begin{aligned}
G_{\mathrm{D}} & \equiv \frac{I_{1 / 3, u}-I_{1 / 3, l}}{V_{0}}=\frac{I_{c, R}+I_{1 / 3, u}}{V_{0}} \\
& =\frac{2 e^{2}}{3 h} \frac{\left(1-P_{l, L} P_{u, L}\right)\left(1-P_{l, R} P_{u, R}\right)}{2-P_{l, L} P_{u, L}-P_{l, R} P_{u, R}} .
\end{aligned}
$$

When $P=P_{l, L}=P_{l, R}=P_{u, L}=P_{u, R}=1$, i.e., all the neutral excitations eventually decay $\left(\ell_{\text {ch-eq, } 0} \ll L_{\text {arm }}\right)$, the conductance can be seen to be zero up to an exponential correction of $\sim \exp \left(-L_{\text {arm }} / \ell_{\text {ch-eq, } 0}\right)$ as $1-P$ goes as $1-P \sim$ $\exp \left(-L_{\text {arm }} / \ell_{\text {ch-eq, } 0}\right)$. When all the tunneling probabilities, on the other hand, go to zero, the conductance becomes $e^{2} /(3 h)$.

\section{APPENDIX F: NONEQUILIBRIUM NOISE}

In this Appendix, the noise of neutral currents is shown to be converted into the noise of charge currents by Eq. (25) when all neutral excitations decay $\left(\ell_{\text {ch-eq }, 0} \ll L_{\text {arm }}\right)$. All the calculations in this Appendix are performed employing model B.

The densities of neutral modes $n_{1}$ and $n_{2}$ are defined as $\rho_{n_{1}}(x)=\partial_{x} \phi_{n_{1}} /(\sqrt{2} \pi)$ and $\rho_{n_{2}}=3 \partial_{x} \phi_{n_{2}} /(\sqrt{2} \pi)$ such that creation of an up neutralon changes the density of neutral mode $n_{1}$ and $n_{2}$ by a delta function contribution since

$$
\begin{gathered}
\psi_{u}\left(x^{\prime}\right)\left(\frac{\partial_{x} \phi_{n_{1}}(x)}{\sqrt{2} \pi}\right) \psi_{u}^{\dagger}\left(x^{\prime}\right)=\frac{\partial_{x} \phi_{n_{1}}(x)}{\sqrt{2} \pi}+\delta\left(x-x^{\prime}\right), \\
\psi_{u}\left(x^{\prime}\right)\left(\frac{3 \partial_{x} \phi_{n_{2}}(x)}{\sqrt{2} \pi}\right) \psi_{u}^{\dagger}\left(x^{\prime}\right)=\frac{3 \partial_{x} \phi_{n_{2}}(x)}{\sqrt{2} \pi}+\delta\left(x-x^{\prime}\right) .
\end{gathered}
$$

Then, we define decay neutral current operators $I_{\mathrm{dec}, n_{1}}$ and $I_{\mathrm{dec}, n_{2}}$ in the decay region of neutralons from the equations of motion of neutral number operators $N_{n_{1}}=\int d x \rho_{n_{1}}(x)$ and $N_{n_{2}}=\int d x \rho_{n_{2}}(x)$ as

$$
\begin{aligned}
I_{\mathrm{dec}, n_{1}} & =\frac{d N_{n_{1}}}{d t}=\frac{i}{\hbar}\left[H_{\mathrm{eq}}, N_{n_{1}}\right] \\
& =-\frac{i}{h a} \sum_{\epsilon= \pm} \int d x \epsilon\left[T_{u}(x)-T_{d}(x)\right]^{\epsilon}, \\
I_{\mathrm{dec}, n_{2}} & =\frac{d N_{n_{2}}}{d t}=\frac{i}{\hbar}\left[H_{\mathrm{eq}}, N_{n_{2}}\right] \\
& =-\frac{i}{h a} \sum_{\epsilon= \pm} \int d x \epsilon\left[T_{u}(x)+T_{d}(x)-2 T_{s}(x)\right]^{\epsilon},
\end{aligned}
$$

where the tunneling operator $T_{j=u, d, s}$ of each neutralon is defined as

$$
T_{j}(x)=t_{j}(x) \psi_{j}^{\dagger}(x) e^{-i \phi_{c}(x) / \sqrt{3}} e^{i \phi_{1 / 3}(x)}
$$

and a convenient notation $[A B]^{+}=A B\left([A B]^{-}=B^{\dagger} A^{\dagger}\right)$ is used. Similarly, a charge tunneling current is defined as

$$
\begin{aligned}
I_{\text {tun }} & =\frac{i e}{\hbar}\left[H_{\mathrm{eq}}, \frac{1}{2 \pi} \int d x \partial_{x} \phi_{1 / 3}\right] \\
& =\frac{i e}{3 h a} \sum_{\epsilon= \pm} \sum_{j=u, d, s} \int d x \epsilon\left[T_{u}(x)+T_{d}(x)+T_{s}(x)\right]^{\epsilon} .
\end{aligned}
$$

The decay neutral currents and incoming neutral currents are zero, $\left\langle I_{\mathrm{dec}, n_{1}}\right\rangle=\left\langle I_{\mathrm{dec}, n_{2}}\right\rangle=0$ and $\left\langle I_{n_{1}}\right\rangle=\left\langle I_{n_{2}}\right\rangle=0$ [for their definitions, see the in-line equations just below Eq. (23)]; $\left\langle I_{\mathrm{dec}, n_{1}}\right\rangle=\left\langle I_{\mathrm{dec}, n_{2}}\right\rangle=0$ can be derived using the fact that the Green's function of neutralons [Eq. (20)] is real for model B. $\left\langle I_{n_{1}}\right\rangle=\left\langle I_{n_{2}}\right\rangle=0$ can be derived (i) taking the first derivative of Eqs. (21) and (22) by $\lambda_{1}$ and $\lambda_{2}$ and (ii) sending $\lambda_{1}$ and $\lambda_{2}$ to zero. Furthermore, the electrical tunneling current is zero as seen in Eq. (20). Under the assumption that all neutral excitations eventually decay in the decay region, the noise $\left(S_{n_{1}}\right.$ and $S_{n_{2}}$ ) of incoming neutral currents is identical to the noise of the decay neutral currents,

$$
S_{n_{1}}=S_{\mathrm{dec}, \mathrm{n}_{1}} \equiv 2 \int_{-\infty}^{\infty} d t\left\langle I_{\mathrm{dec}, n_{1}}(t) I_{\mathrm{dec}, n_{1}}(0)\right\rangle, \quad S_{n_{2}}=S_{\mathrm{dec}, \mathrm{n}_{2}} \equiv 2 \int_{-\infty}^{\infty} d t\left\langle I_{\mathrm{dec}, n_{2}}(t) I_{\mathrm{dec}, n_{2}}(0)\right\rangle
$$

respectively. Using Eqs. (F2)-(F5), the noise $\left(S_{\text {tun }}\right)$ of the electrical tunneling current can be decomposed into the noise of the neutral decay currents as

$$
\begin{aligned}
S_{\text {tun }} & \equiv 2 \int d t\left\langle I_{\text {tun }}(t) I_{\text {tun }}(0)\right\rangle \\
& \simeq \frac{e^{2}}{9 \hbar^{2} \pi a} \sum_{\epsilon= \pm} \int d t \int d x \int d x^{\prime}\left(\left\langle\left[T_{s}(x, t)\right]^{\epsilon}\left[T_{s}^{\dagger}\left(x^{\prime}, 0\right)\right]^{\epsilon}\right\rangle+\left\langle\left[T_{u}(x, t)\right]^{\epsilon}\left[T_{u}^{\dagger}\left(x^{\prime}, 0\right)\right]^{\epsilon}\right\rangle+\left\langle\left[T_{d}(x, t)\right]^{\epsilon}\left[T_{d}^{\dagger}\left(x^{\prime}, 0\right)\right]^{\epsilon}\right\rangle\right) \\
& =\frac{e^{2}}{9 \hbar^{2} \pi a} \sum_{\epsilon= \pm} \int d t \int d x \int d x^{\prime}\left[\frac{3}{4}\left(\left\langle\left[T_{u}(x, t)\right]^{\epsilon}\left[T_{u}^{\dagger}\left(x^{\prime}, 0\right)\right]^{\epsilon}\right\rangle+\left\langle\left[T_{d}(x, t)\right]^{\epsilon}\left[T_{d}^{\dagger}\left(x^{\prime}, 0\right)\right]^{\epsilon}\right\rangle\right)\right. \\
& \left.+\frac{1}{4}\left(\left\langle\left[T_{u}(x, t)\right]^{\epsilon}\left[T_{u}^{\dagger}\left(x^{\prime}, 0\right)\right]^{\epsilon}\right\rangle+\left\langle\left[T_{d}(x, t)\right]^{\epsilon}\left[T_{d}^{\dagger}\left(x^{\prime}, 0\right)\right]^{\epsilon}\right\rangle+4\left\langle\left[T_{s}(x, t)\right]^{\epsilon}\left[T_{s}^{\dagger}\left(x^{\prime}, 0\right)\right]^{\epsilon}\right\rangle\right)\right] \\
& =\frac{e^{2}}{36}\left(3 S_{\mathrm{dec}, n_{1}}+S_{\mathrm{dec}, n_{2}}\right)=\frac{e^{2}}{36}\left(3 S_{n_{1}}+S_{n_{2}}\right) .
\end{aligned}
$$


This charge noise of the electrical tunneling current is measured at drain D (see Fig. 2). The charge noise in each of the upper and lower edges contributes to the zero-frequency noise $S_{\mathrm{D}}$ as

$$
S_{\mathrm{D}}=\frac{e^{2}}{36}\left(3 S_{n_{1}, \mathrm{u}}+S_{n_{2}, \mathrm{u}}+3 S_{n_{1}, 1}+S_{n_{2}, 1}\right)=\frac{2 e^{3} V}{9 h},
$$

arriving at Eq. (25).

[1] X.-G. Wen, Theory of the edge states in fractional quantum Hall effects, Int. J. Mod. Phys. B 6, 1711 (1992).

[2] A. Bid, N. Ofek, H. Inoue, M. Heiblum, C. L. Kane, V. Umansky, and D. Mahalu, Observation of neutral modes in the fractional quantum Hall regime, Nature (London) 466, 585 (2010).

[3] I. Gurman, R. Sabo, M. Heiblum, V. Umansky, and D. Mahalu, Extracting net current from an upstream neutral mode in the fractional quantum Hall regime, Nat. Commun. 3, 1289 (2012).

[4] Y. Gross, M. Dolev, M. Heiblum, V. Umansky, and D. Mahalu, Upstream Neutral Modes in the Fractional Quantum Hall Effect Regime: Heat Waves or Coherent Dipoles, Phys. Rev. Lett. 108, 226801 (2012).

[5] V. Venkatachalam, S. Hart, L. Pfeiffer, K. West, and A. Yacoby, Local thermometry of neutral modes on the quantum Hall edge, Nat. Phys. 8, 676 (2012).

[6] C. Altimiras, H. le Sueur, U. Gennser, A. Anthore, A. Cavanna, D. Mailly, and F. Pierre, Chargeless Heat Transport in the Fractional Quantum Hall Regime, Phys. Rev. Lett. 109, 026803 (2012).

[7] H. Inoue, A. Grivnin, Y. Ronen, M. Heiblum, V. Umansky, and D. Mahalu, Proliferation of neutral modes in fractional quantum Hall states, Nat. Commun. 5, 4067 (2014).

[8] S. Takei and B. Rosenow, Neutral mode heat transport and fractional quantum Hall shot noise, Phys. Rev. B 84, 235316 (2011).

[9] G. Viola, S. Das, E. Grosfeld, and A. Stern, Thermoelectric Probe for Neutral Edge Modes in the Fractional Quantum Hall Regime, Phys. Rev. Lett. 109, 146801 (2012).

[10] O. Shtanko, K. Snizhko, and V. Cheianov, Nonequilibrium noise in transport across a tunneling contact between $v=\frac{2}{3}$ fractional quantum Hall edges, Phys. Rev. B 89, 125104 (2014).

[11] A. Bid, N. Ofek, M. Heiblum, V. Umansky, and D. Mahalu, Shot Noise and Charge at the 2/3 Composite Fractional Quantum Hall State, Phys. Rev. Lett. 103, 236802 (2009).

[12] S. Takei, B. Rosenow, and A. Stern, Noise due to neutral modes in the $v=2 / 3$ fractional quantum Hall state, Phys. Rev. B 91, 241104(R) (2015).

[13] R. Sabo, I. Gurman, A. Rosenblatt, F. Lafont, D. Banitt, J. Park, M. Heiblum, Y. Gefen, V. Umansky, and D. Mahalu, Edge reconstruction in fractional quantum Hall states, Nat. Phys. 13, 491 (2017).

[14] A. Rosenblatt, F. Lafont, I. Levkivskyi, R. Sabo, I. Gurman, D. Banitt, M. Heiblum, and V. Umansky, Transmission of heat modes across a potential barrier, Nat. Commun. 8, 2251 (2017).

[15] S. K. Srivastav, M. R. Sahu, K. Watanabe, T. Taniguchi, S. Banerjee, and A. Das, Universal quantized thermal conductance in graphene, Sci. Adv. 5, eaaw5798 (2019).

[16] D. B. Chklovskii, B. I. Shklovskii, and L. I. Glazman, Electrostatics of edge channels, Phys. Rev. B 46, 4026 (1992).
[17] J. Dempsey, B. Y. Gelfand, and B. I. Halperin, ElectronElectron Interactions and Spontaneous Spin Polarization in Quantum Hall Edge States, Phys. Rev. Lett. 70, 3639 (1993).

[18] C. de C. Chamon and X. G. Wen, Sharp and smooth boundaries of quantum Hall liquids, Phys. Rev. B 49, 8227 (1994).

[19] Y. Meir, Composite Edge States in the $v=2 / 3$ Fractional Quantum Hall Regime, Phys. Rev. Lett. 72, 2624 (1994).

[20] J. Wang, Y. Meir, and Y. Gefen, Edge Reconstruction in the $v=2 / 3$ Fractional Quantum Hall State, Phys. Rev. Lett. 111, 246803 (2013).

[21] A. H. MacDonald, Edge States in the Fractional-Quantum-HallEffect Regime, Phys. Rev. Lett. 64, 220 (1990).

[22] S. L. Sondhi, A. Karlhede, S. A. Kivelson, and E. H. Rezayi, Skyrmions and the crossover from the integer to fractional quantum Hall effect at small Zeeman energies, Phys. Rev. B 47, 16419 (1993).

[23] K. Yang, Field Theoretical Description of Quantum Hall Edge Reconstruction, Phys. Rev. Lett. 91, 036802 (2003).

[24] X. Wan, E. H. Rezayi, and K. Yang, Edge reconstruction in the fractional quantum Hall regime, Phys. Rev. B 68, 125307 (2003).

[25] X. Wan, K. Yang, and E. H. Rezayi, Reconstruction of Fractional Quantum Hall Edges, Phys. Rev. Lett. 88, 056802 (2002).

[26] U. Khanna, M. Goldstein, and Y. Gefen, Fractional edge reconstruction in integer quantum Hall phases, Phys. Rev. B 103, L121302 (2021).

[27] C. L. Kane and M. P. A. Fisher, Quantized thermal transport in the fractional quantum Hall effect, Phys. Rev. B 55, 15832 (1997).

[28] C. L. Kane, M. P. A. Fisher, and J. Polchinski, Randomness at the Edge: Theory of Quantum Hall Transport at Filling $v=2 / 3$, Phys. Rev. Lett. 72, 4129 (1994).

[29] C. L. Kane and M. P. A. Fisher, Impurity scattering and transport of fractional quantum Hall edge states, Phys. Rev. B 51, 13449 (1995).

[30] B. Rosenow and B. I. Halperin, Signatures of neutral quantum Hall modes in transport through low-density constrictions, Phys. Rev. B 81, 165313 (2010).

[31] I. V. Protopopov, Y. Gefen, and A. D. Mirlin, Transport in a disordered $v=2 / 3$ fractional quantum Hall junction, Ann. Phys. (Amsterdam) 385, 287 (2017).

[32] C. Nosiglia, J. Park, B. Rosenow, and Y. Gefen, Incoherent transport on the $v=2 / 3$ quantum Hall edge, Phys. Rev. B 98, 115408 (2018).

[33] Y. Cohen, Y. Ronen, W. Yang, D. Banitt, J. Park, M. Heiblum, A. D. Mirlin, Y. Gefen, and V. Umansky, Synthesizing a $v=2 / 3$ fractional quantum Hall effect edge state from counterpropagating $v=1$ and $v=1 / 3$ states, Nat. Commun. 10, 1920 (2019).

[34] R. Bhattacharyya, M. Banerjee, M. Heiblum, D. Mahalu, and V. Umansky, Melting of Interference in the Fractional Quantum 
Hall Effect: Appearance of Neutral Modes, Phys. Rev. Lett. 122, 246801 (2019).

[35] Reference [13] includes a phenomenological analysis to account for the shot noise on the conductance plateau. However, a theoretical study based on microscopic dynamics is still lacking.

[36] J. Park, A. D. Mirlin, B. Rosenow, and Y. Gefen, Noise on complex quantum Hall edges: Chiral anomaly and heat diffusion, Phys. Rev. B 99, 161302(R) (2019).

[37] C. Spånslätt, J. Park, Y. Gefen, and A. D. Mirlin, Topological Classification of Shot Noise on Fractional Quantum Hall Edges, Phys. Rev. Lett. 123, 137701 (2019).

[38] C. Spånslätt, J. Park, Y. Gefen, and A. D. Mirlin, Conductance plateaus and shot noise in fractional quantum Hall point contacts, Phys. Rev. B 101, 075308 (2020).

[39] T. Cheng and L. Li, Gauge Theory of Elementary Particle Physics (Oxford University Press, Oxford, 1994).
[40] D. B. Gutman, Y. Gefen, and A. D. Mirlin, Nonequilibrium Luttinger Liquid: Zero-Bias Anomaly and Dephasing, Phys. Rev. Lett. 101, 126802 (2008).

[41] I. P. Levkivskyi and E. V. Sukhorukov, Noise-Induced Phase Transition in the Electronic Mach-Zehnder Interferometer, Phys. Rev. Lett. 103, 036801 (2009).

[42] D. B. Gutman, Y. Gefen, and A. D. Mirlin, Bosonization of one-dimensional fermions out of equilibrium, Phys. Rev. B 81, 085436 (2010).

[43] I. P. Levkivskyi and E. V. Sukhorukov, Energy relaxation at quantum Hall edge, Phys. Rev. B 85, 075309 (2012).

[44] B. Rosenow, I. P. Levkivskyi, and B. I. Halperin, Current Correlations from a Mesoscopic Anyon Collider, Phys. Rev. Lett. 116, 156802 (2016).

[45] I. P. Levkivskyi, Universal nonequilibrium states at the fractional quantum Hall edge, Phys. Rev. B 93, 165427 (2016). 